Testes de hipóteses agnósticos como alternativa logicamente consistente em testes simultâneos

Bernardo Franco Reimann

\author{
DisSERTAÇÃO APRESENTADA \\ $A O$ \\ Instituto de Matemática e EstatísticA \\ DA \\ Universidade DE SÃo PAUlo \\ PARA \\ OBTENÇÃO DO TÍTULO \\ $\mathrm{DE}$ \\ Mestre em CiênCIAS \\ Programa: Estatística \\ Orientador: Prof. Dr. Luís Gustavo Esteves
}

São Paulo, julho de 2020 


\section{Testes de hipóteses agnósticos como alternativa logicamente consistente em testes simultâneos}

Esta versão da dissertação contém as correções e alterações sugeridas pela Comissão Julgadora durante a defesa da versão original do trabalho, realizada em 01/07/2020. Uma cópia da versão original está disponível no

Instituto de Matemática e Estatística da Universidade de São Paulo.

Comissão Julgadora:

- Prof. Dr. Luís Gustavo Esteves (orientador) - IME-USP

- Prof. Dr. Rafael Bassi Stern - UFSCar

- Prof ${ }^{a}$. Dra . Veronica Andréa González-Lopez - UNICAMP 


\section{Resumo}

Reimann, B. F. Testes de hipóteses agnósticos como alternativa logicamente consistente em testes simultâneos. 2020. 30 f. Dissertação (Mestrado) - Instituto de Matemática e Estatística, Universidade de São Paulo, São Paulo, 2020.

Testes de hipóteses são uma ferramenta da inferência estatística muito utilizada por profissionais de diversas áreas. Infelizmente, ainda que testes ótimos tenham sido construídos para a avaliação de uma única hipótese, é possível obter conclusões difíceis de interpretar ao realizarmos testes simultâneos com o mesmo conjunto de dados. Diversos estudos recentes discutem propriedades que caracterizam possíveis inconsistências lógicas ao testar hipóteses distintas simultaneamente. Nesse trabalho, revisamos essas propriedades com exemplos para os testes de hipóteses usuais. Também revisamos essas propriedades para os testes de hipóteses agnósticos, onde, alem de aceitar ou rejeitar uma determinada hipótese, podemos permanecer agnósticos, suspendendo o julgamento nos casos em que a evidência fornecida pelos dados for insuficiente para decidir pelas duas alternativas usuais. Finalmente, apresentamos ainda alguns resultados que conferem otimalidade estatística, sob a perspectiva da teoria da decisão bayesiana, a testes agnósticos logicamente consistentes.

Palavras-chave: testes agnósticos, testes de hipóteses, testes simultâneos, teoria da decisão, propriedades lógicas. 


\section{Abstract}

Reimann, B. F. Agnostic hypothesis testing as a logically consistent alternative in simultaneous tests. 2020. 30 f. Dissertação (Mestrado) - Instituto de Matemática e Estatística, Universidade de São Paulo, São Paulo, 2020.

Hypothesis tests are a statistical inference tool widely used by professionals in different areas. Unfortunately, it is possible to obtain conclusions that are diffcult to interpret when performing simultaneous tests with the same data set despite the existence of optimal tests for a single hypothesis. Several recent studies discuss properties that characterize possible logical inconsistencies when testing different hypotheses simultaneously. In this work we review these properties with examples for the usual hypothesis tests. We also review these properties for agnostic hypothesis tests, in which, in addition to accepting or rejecting a given hypothesis, it is possible to remain agnostic, withholding judgment in cases where the evidence provided by the data is insuficient to decide between the two usual alternatives. Finally, we present some results that grant statistical optimality to agnostic hypothesis testing, under the Bayesian decision-theoretic framework.

Keywords: agnostic tests, hypothesis testing, simultaneous tests, decision theory, logical properties. 


\section{Sumário}

1 Introdução $\quad \mathbf{1}$

1.1 Considerações Iniciais $\ldots \ldots \ldots \ldots \ldots \ldots \ldots \ldots$

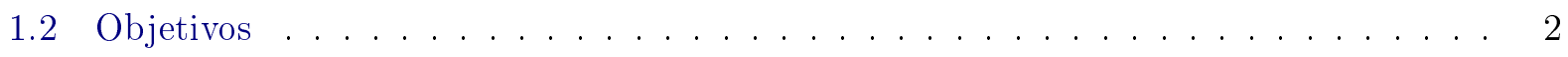

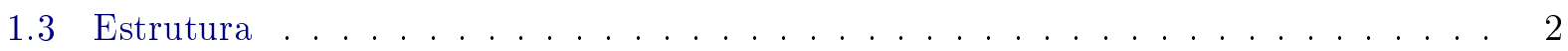

2 Propriedades Lógicas de Testes de Hipóteses $\quad 3$

2.1 Definições Iniciais . . . . . . . . . . . . . . . . . . . . . . . . . . . . . . . . . . . . . . . . . . . . .

2.2 Monotonicidade . . . . . . . . . . . . . . . . . . . . 4

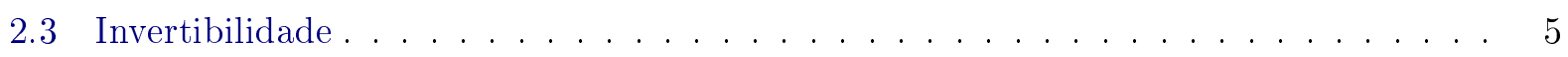

2.4 Consonância da União . . . . . . . . . . . . . . . . . . . . . . 6

2.5 Consonância da Intersecção . . . . . . . . . . . . . . . . . . . . . 7

2.6 Propriedades em Conjunto . . . . . . . . . . . . . . . . . . 8

3 Testes de Hipóteses Agnósticos $\quad 11$

3.1 Definições Iniciais . . . . . . . . . . . . . . . . . . . . . . . 11

3.2 Propriedades Lógicas nos Testes de Hipóteses Agnósticos . . . . . . . . . . . . . . . . 13

3.2 .1 Monotonicidade . . . . . . . . . . . . . . . . . . . 13

3.2 .2 Invertibilidade . . . . . . . . . . . . . . . . . . . . 14

3.2 .3 Consonância da União . . . . . . . . . . . . . . . . . . . . . . . 15

3.2 .4 Consonância da Intersecção . . . . . . . . . . . . . . . . . . . . . 16

3.3 Testes de Hipóteses Agnósticos Consistentes . . . . . . . . . . . . . . . . . . . . . . . . . . . . . . . . . . . . . 17

3.3 .1 Consistência Fraca . . . . . . . . . . . . . . . . . . . . 17

3.3 .2 Consistência Forte . . . . . . . . . . . . . . . . . . 19

3.4 Resultados Considerando a Teoria da Decisão . . . . . . . . . . . . . . . . . . . 20

4 Conclusões $\quad 27$

4.1 Considerações Finais . . . . . . . . . . . . . . . . . . . 27

4.2 Perspectivas Futuras . . . . . . . . . . . . . . . 27

$\begin{array}{ll}\text { Referências Bibliográficas } & 29\end{array}$ 


\section{Capítulo 1}

\section{Introdução}

\subsection{Considerações Iniciais}

Na inferência estatística, estamos interessados em utilizar um conjunto de dados observados para fazer afirmações a respeito de sua distribuição ou de seu parâmetro $\theta$ (Lehmann e Romano (2005)). Dentro desse contexto, os testes de hipóteses permitem avaliar se uma distribuição ou um conjunto de possíveis distribuições são plausíveis, considerando os dados observados (Kendall e Stuart (1961)).

Em diversas aplicações, podemos estar interessados em testar mais de uma hipótese sobre os dados observados. Por exemplo, no modelo usual de análise de variância (ANOVA), é comum testar a igualdade das médias de todos os grupos e também entre pares de grupos. Em pesquisas eleitorais de eleições majoritárias em dois turnos (Nicolau (1993)), podemos testar se algum candidato específico será vencedor em primeiro turno ou se haverá segundo turno.

Algo comum a essas situações, em que realizamos testes de hipóteses simultâneos, é que os testes usuais podem fornecer resultados contraditórios ou difíceis de interpretar: por exemplo, no caso de ANOVA, podemos não ter evidências de que pelo menos uma média dos grupos é diferente das outras mas ter evidências de que algum par de médias, quando comparadas isoladamente, são diferentes; em pesquisas eleitorais, podemos rejeitar a hipótese de que a eleição terá segundo turno mas também rejeitar que todos os candidatos ganhem em primeiro turno, ao testar as hipóteses de vitória separadamente. Essas situações são comuns, mesmo quando utilizamos testes que obedecem os critérios usuais de otimalidade, como os testes uniformemente mais poderosos (UMP), sob a perspectiva frequentista, e os testes de Bayes (Lehmann e Romano (2005)), sob a perspectiva bayesiana.

Dentro desse contexto, alguns trabalhos recentes tem buscado definir propriedades que caracterizem formalmente diferentes tipos de coerência lógica e investigar testes que respeitem-nas, individualmente ou em conjunto, resultando em diferentes níveis de consistência lógica nos resultados, alem de avaliar se apresentam algum nível de otimalidade. Infelizmente, Izbicki (2010) conclui que testes com obediência total a essas propriedades são testes muito simples baseados apenas em estimadores pontuais.

Uma alternativa à abordagem usual são os testes agnósticos, que podem, para alguma hipótese, resultar em uma das três opções: aceitar a hipótese quando os dados fornecerem evidência favorável, rejeitar a hipótese quando os dados fornecerem evidência contrária ou, em casos onde os dados não suportam nenhuma das alternativas, abster-se da escolha entre as duas opções anteriores. 


\subsection{Objetivos}

Os objetivos dessa dissertação são:

- Revisar as propriedades lógicas avaliadas em estudos anteriores para testes de hipóteses simultâneos.

- Revisar o desenvolvimento dos testes agnósticos até o presente momento e sua relação com as propriedades já estudadas.

- Introduzir novos resultados que relacionam testes de hipóteses agnósticos logicamente consistentes, que obedeçam as propriedades revisadas, com a teoria da decisão bayesiana.

\subsection{Estrutura}

No capítulo 2, revisamos as propriedades lógicas já estudadas para os testes de hipóteses usuais, incluindo exemplos de testes que atendem ou não essas propriedades e os tipos de inconsistência lógica decorrentes disso, também revisamos os resultados que estão ligados à exigência dessas propriedades em conjunto. No capítulo 3, trazemos a definição de testes de hipóteses agnósticos e adaptamos as propriedades vistas no capítulo anterior para esse caso, revisamos os tipos de coerência lógica possíveis decorrentes da exigência dessas propriedades simultâneamente e, por último, apresentamos alguns resultados relacionando teoria da decisão bayesiana aos testes de hipóteses agnósticos logicamente consistentes. Encerramos discutindo algumas conclusões e perspectivas futuras no capítulo 4 . 


\section{Capítulo 2}

\section{Propriedades Lógicas de Testes de Hipóteses}

A discussão da existência de inconsistências lógicas em testes de hipóteses simultâneos e formas possíveis de evitá-las não é nova. Gabriel (1969) discute possíveis soluções para casos onde uma hipótese é rejeitada mas hipóteses específicas formadas por subconjuntos de distribuições da primeira não são, quando testadas individualmente. Betz e Levin (1982) discute estruturas de testes para evitar a inconsistência lógica no caso da ANOVA citado na introdução. Outras propostas podem ser encontradas em Rom e Holland (1995) e Hommel e Bretz (2008).

Dentro desse contexto, alguns trabalhos recentes procuraram definir mais formalmente essas propriedades. Silva (2010) define a propriedade de monotonicidade, que também foi estudada em Gabriel (1969) e Fossaluza (2008). Izbicki (2010), além dela, também propõe algumas outras propriedades como invertibilidade, consonância da união e consonância da intersecção. Finalmente, Silva (2014) estuda funções de perda que geram testes de Bayes atendendo essas propriedades uma a uma e em conjunto.

Nesse capítulo, vamos rever as propriedades lógicas estudadas nas referencias citadas, trazendo alguns exemplos de testes que as obedecem e também de casos que não as obedecem. Além disso, também vamos destacar alguns resultados decorrentes da exigência dessas propriedades simultaneamente.

\subsection{Definições Iniciais}

Nessa dissertação, seguiremos a notação utilizada nos trabalhos anteriores citados no inicio do capítulo. Consideraremos $\Theta$ como o espaço paramétrico com $\sigma$-álgebra $\sigma(\Theta)$ e parâmetros $\theta \in \Theta$. Estamos interessados em inferir sobre $\theta$ por meio das possíveis amostras $x$ do espaço amostral $\chi$.

Considerando uma família de distribuições $\mathcal{P}=\left\{\mathbb{P}_{\theta}: \theta \in \Theta\right\}$ para $\chi$, indexada por $\theta$, estamos interessados em avaliar se a amostra observada $x$ é compatível com o conjunto de distribuições $\mathcal{P}_{0}=\left\{\mathbb{P}_{\theta_{0}}: \theta_{0} \in \Theta_{0}\right\}$, induzido pelo conjunto de parâmetros $\Theta_{0} \subseteq \Theta$, nossa hipótese. Essa hipótese é denominada a hipótese nula, e $\Theta-\Theta_{0}=\Theta_{1}$ é a hipótese alternativa. Utilizaremos uma função de teste $\phi: \chi \rightarrow\{0,1\}$ que associa a cada amostra possível $x$ a decisão de rejeitar a hipótese, representada por $1(\phi(x)=1)$, quando os dados não trouxerem evidência favorável, ou a decisão de aceitar a hipótese, representada por $0(\phi(x)=0)$, quando trouxerem. 
Um detalhe importante é que aqui estamos considerando um teste de hipóteses como um problema de decisão com duas alternativas (Lindgren (1993)): aceitar a hipótese (comportar-se como se $\theta$ estivesse em $\Theta_{0}$ ) ou rejeitar (comportar-se como se $\theta$ não estivesse em $\Theta_{0}$ e, portanto, no complementar com respeito a $\Theta$ ). Também não fazemos distinção entre as expressões: "não rejeitar a hipótese" e "aceitar a hipótese", de modo que usamos esses termos apenas como identificação de um dos resultados para a conclusão do teste frente aos dados, como discutido em Kruskal (1978) e Kendall e Stuart (1961).

Finalmente, como podemos estar interessados em testar várias hipóteses a partir de uma amostra observada, precisamos de um objeto para associar os testes à cada hipótese possível. Para isso, utilizamos o conceito de classe de teste de hipóteses:

Definição 2.1 (Classe de testes de hipóteses - Izbicki (2010)). Uma classe de testes de hipóteses pode ser definida como uma função $\mathcal{L}: \sigma(\Theta) \rightarrow \Phi$, onde $\sigma(\Theta)$ é uma $\sigma$-álgebra do espaço paramétrico $e \Phi=\{\phi: \chi \rightarrow\{0,1\}\}$ é o conjunto de todas as funçôes de testes possíveis. Dessa forma, $\mathcal{L}$ associa a cada possivel hipótese $A \in \sigma(\Theta)$ uma função de teste para avaliar $H: \theta \in A$ na amostra $x$ observada. Podemos também representar a classe por meio das funçóes de testes indexadas pelos conjuntos de $\sigma(\Theta),\left(\phi_{A}\right)_{A \in \sigma(\Theta)}$

Nas próximas seções desse capítulo, relembramos algumas propriedades lógicas que classes de testes de hipóteses podem apresentar.

\subsection{Monotonicidade}

Classes de testes com a propriedade de monotonicidade são aquelas onde a aceitação de uma hipótese implica na aceitação de todas as outras hipóteses que contém a primeira. Essa propriedade foi chamada de coerência em Gabriel (1969) e foi estudada mais recentemente em Fossaluza (2008) no contexto de pesquisas eleitorais. Além disso, Izbicki (2010) avalia essa propriedade em diversas classes de testes frequentemente utilizadas. Silva (2010) e Silva (2014) também avaliam essa propriedade sobre a ótica da teoria da decisão bayesiana, caracterizando funções de perdas que geram testes de Bayes com essa propriedade. Formalmente, temos a seguinte definição:

Definição 2.2 (Monotonicidade - Izbicki (2010)). Uma classe de testes $\mathcal{L}$ atende a propriedade de monotonicidade (é monotônica), se, $\forall A, B \in \sigma(\Theta), A \subseteq B \Rightarrow \mathcal{L}(A) \geq \mathcal{L}(B)$.

Ou seja, para uma amostra $x$ observada, se uma hipótese $A$ for aceita $(\mathcal{L}(A)(x)=0)$ todas as hipóteses que contém $A$ deverão ser aceitas $(\mathcal{L}(B)(x)=0, \forall B \supseteq A)$. A seguir, apresentamos um exemplo de classe de testes que atende essa propriedade:

Exemplo 2.1 (Classe de testes FBST - Izbicki (2010)). Seja $\Theta=\mathbb{R}^{n}, \sigma(\Theta)=\mathcal{B}\left(\mathbb{R}^{n}\right)$ e $f(\theta)$ uma distribuição a priori para $\theta$ onde, para todo $x \in \chi$ existe $f(\theta \mid x)$ a função densidade a posteriori de $\theta$ dado $x$ observado. Para cada hipótese $A \in \sigma(\Theta)$ e $x \in \chi$, definimos $T_{x}^{A}=\{\theta \in \Theta: f(\theta \mid x)>$ $\left.\sup _{A} f(\theta \mid x)\right\}$ o conjunto tangente à hipótese nula e $e v_{x}(A)=1-P\left(\theta \in T_{x}^{A} \mid x\right)$ o valor de evidência de Pereira-Stern (Pereira e Stern (1999)) para a hipótese $A$. Definimos uma classe de testes $\mathcal{L}$ onde aceitamos a hipótese de interesse quando sua medida de evidência for alta:

$$
\mathcal{L}(A)(x)=\left\{\begin{array}{ll}
1, & \text { se } e v_{x}(A) \leq c \\
0, & \text { caso contrário }
\end{array}, \quad c \in[0,1]\right.
$$


Essa classe de testes é monotônica. Com efeito, para $A, B \in \sigma(\Theta)$ com $A \subseteq B$, temos que $\sup _{B} f(\theta \mid x) \geq \sup _{A} f(\theta \mid x)$ e portanto $T_{x}^{B} \subseteq T_{x}^{A}$ e $e v_{x}(A) \leq e v_{x}(B)$. Então, para $x \in \chi$ tal que $\mathcal{L}(A)(x)=0$ :

$$
\mathcal{L}(A)(x)=0 \Rightarrow e v_{x}(A)>c \Rightarrow e v_{x}(B)>c \Rightarrow \mathcal{L}(B)(x)=0
$$

Nem todos os testes atendem essa propriedade, em particular, nos casos onde estamos interessados nos testes mais poderosos, que são os testes frequentistas usuais, ao avaliarmos $\theta$, se pensarmos em hipóteses alternativas bilaterais e unilaterais, essa propriedade não é atendida. Além disso, os testes baseados em níveis de significância (p-valores) também não atendem essa propriedade, como podemos ver no próximo exemplo.

Exemplo 2.2 (Nível de significância - Royall (1997)). Seja $X$ uma variável aleatória binomial com parâmetros $(n, \theta), \chi=\{0,1,2, \ldots, n\}, \Theta=[0,1]$, e considere $\mathcal{L}$ a classe de testes que rejeita a hipótese quando o nível de significância (p-valor) for menor que $\alpha$.

Ao testarmos a hipótese $\theta=\frac{1}{2}$, para um valor $x$ observado, se $x \geq \frac{n}{2}$, o teste se baseia em rejeitar a hipótese se $P(X \geq x) \leq \frac{\alpha}{2}$. Por outro lado, ao considerarmos a hipótese $\theta \leq \frac{1}{2}$, o teste se baseia em rejeitar a hipótese se $P(X \geq x) \leq \alpha$. De modo que, se observarmos um valor de $x$ tal que $\frac{\alpha}{2}<\mathbb{P}(X \geq x) \leq \alpha$ decidiríamos por não rejeitar a hipótese $\theta=\frac{1}{2}$ mas rejeitamos a hipótese maior $\theta \leq \frac{1}{2}$. Essa classe, portanto, não atende a propriedade de monotonicidade.

\subsection{Invertibilidade}

Outra propriedade interessante em testes simultâneos é a de invertibilidade, essa propriedade descreve um comportamento que é comum nos testes de hipóteses bayesianos, mas em geral não é respeitada nos testes frequentistas. Dizemos que uma classe de testes atende a propriedade de invertibilidade se a hipótese nula pode ser trocada pela alternativa e a conclusão do teste a respeito dos parâmetros permanece a mesma, mais formalmente:

Definição 2.3 (Invertibilidade - Izbicki (2010)). Uma classe de testes $\mathcal{L}$ atende a propriedade de invertibilidade (é invertivel), se, $\forall A \in \sigma(\Theta), \mathcal{L}(A)=1-\mathcal{L}\left(A^{c}\right)$.

Ou seja, para qualquer hipótese $A$, sua rejeição implica na aceitação da hipótese alternativa $A^{c}$ quando testada e vice-versa. Sob a abordagem clássica, os testes uniformemente mais poderosos (UMP), por exemplo, em geral não têm essa propriedade: para uma hipótese nula fixada, é de interesse maximizar o poder do teste com um nível de erro do tipo I (rejeitar a hipótese nula quando esta é verdadeira) fixado. Isso acaba gerando probabilidades de erro do tipo II (não rejeitar a hipótese nula quando esta é falsa) maiores, e portanto, uma assimetria entre as duas hipóteses (Robert (2007)). Abaixo traremos um exemplo de teste onde essa propriedade é atendida. Para isso, primeiramente, vamos introduzir o conceito de uma classe de testes de hipóteses gerada por uma família de funções de perda, que permite a construção de testes bayesianos simultâneos.

Definição 2.4 (Classe de testes de Bayes - Silva (2014)). Considere $\left(L_{A}\right)_{A \in \sigma(\Theta)}$ uma familia de funções de perda que para cada hipótese possivel $A \in \sigma(\Theta)$ quantifica os acertos e erros das decisôes de rejeitar ou aceitar a hipótese, $L_{A}:\{0,1\} \times \Theta \rightarrow \mathbb{R}^{+}$. Seja $\pi$ a nossa distribuição a priori em $\Theta$. Podemos definir uma classe de testes $\mathcal{L}$ que liga cada hipótese $A \in \sigma(\Theta)$ ao teste de Bayes com respeito a priori $\pi$ considerando a penalidade $L_{A}$. 
Para essa classe de testes, Silva (2014) explora quais restrições devem ser impostas à família de funções de perda $\left\{L_{A}, A \in \sigma(\Theta)\right\}$ para que os testes gerados por ela respeitem essa propriedade. Em particular, trazemos um exemplo onde essa propriedade é atendida:

Exemplo 2.3 (Classe de testes de Bayes com perda simples ou 0-1). Para a família de funções de perda $\left(L_{A}\right)_{A \in \sigma(\Theta)}$ onde

$$
L_{A}\left(0, \theta_{0}\right)=L_{A}\left(1, \theta_{1}\right)=0 \text { e } L_{A}\left(0, \theta_{1}\right)=L_{A}\left(1, \theta_{0}\right)=1, \theta_{0} \in A, \theta_{1} \notin A
$$

Consideremos a classe de testes de Bayes gerada por essas perdas. Para qualquer $A$ e $x$ os testes se baseiam em rejeitar a hipótese $A$ quando

$$
\mathbb{P}(\theta \in A \mid x) \leq \frac{1}{2}
$$

Dessa forma, como $\mathbb{P}(\theta \notin A \mid x)=1-\mathbb{P}(\theta \in A \mid x)$, essa classe de testes atende a propriedade de invertibilidade.

Vale ressaltar que mesmo para funções de perda mais gerais, como a função de perda 0-1-c (Schervish (1995)), isto é, $L_{A}\left(0, \theta_{0}\right)=L_{A}\left(1, \theta_{1}\right)=0, L_{A}\left(0, \theta_{1}\right)=1$ e $L_{A}\left(1, \theta_{0}\right)=c$ para $\theta_{0} \in A$ e $\theta_{1} \notin A$, o teste segue atendendo essa propriedade desde que as penalidades associadas aos erros de tipo I e tipo II quando a hipótese nula é $A(c$ e 1$)$, coincidam, respectivamente, com as penalidades associadas aos erros de tipo II e de tipo I ao testar a hipótese $A^{c}$, isto é, desde que $L_{A^{c}}\left(0, \theta_{0}\right)=$ $L_{A^{c}}\left(1, \theta_{1}\right)=0, L_{A^{c}}\left(0, \theta_{1}\right)=c$ e $L_{A^{c}}\left(1, \theta_{0}\right)=1$ para $\theta_{0} \in A^{c}$ e $\theta_{1} \notin A^{c}$. A classe de testes do exemplo anterior também atende a propriedade de monotonicidade. Abaixo, trazemos um exemplo de teste UMP onde a propriedade de invertibilidade não é atendida:

Exemplo 2.4 (Teste UMP para proporção de uma distribuição binomial). Seja $X$ uma variável aleatória binomial com parâmetros $(n, \theta), \chi=\{0,1,2,3, \ldots, 10\}, \Theta=[0,1]$. Ao testarmos a hipótese $\theta \leq \frac{1}{2}$, o teste uniformemente mais poderoso ao nível $\alpha$ se baseia em rejeitar a hipótese para um valor $x$ observado, se $\mathbb{P}(X \geq x) \leq \alpha$. Por outro lado, para a hipótese $\theta>\frac{1}{2}$, o teste se baseia em rejeitar a hipótese se $\mathbb{P}(X \leq x) \leq \alpha$.

Dessa forma, é possível observar um valor de $x$ tal que $\mathbb{P}(X \leq x)>\alpha$ e $\mathbb{P}(X \geq x)>\alpha$. Nesse caso, não rejeitaríamos nenhuma das hipóteses e, portanto, esse teste não atende a propriedade de invertibilidade.

\subsection{Consonância da União}

Essa propriedade afirma que se aceitarmos uma hipótese, ao particionarmos tal hipótese em dois conjuntos (isto é, em duas hipóteses cuja união corresponde à hipótese aceita) e ao testarmos essas hipóteses da partição separadamente, ao menos uma delas deverá ser aceita. De fato, parece não fazer sentido afirmar que nossos dados são incompatíveis com dois conjuntos de distribuições quando avaliados separadamente e, ao mesmo tempo, afirmar que são compatíveis com o conjunto de distribuições formado pela união dos conjuntos anteriores.

Definição 2.5 (Consonância da união finita - Izbicki (2010)). Uma classe de testes $\mathcal{L}$ atende a propriedade de consonância da união finita se, $\forall A, B \in \sigma(\Theta), \mathcal{L}(A \cup B) \geq \mathcal{L}(A) \mathcal{L}(B)$. 
Em Izbicki (2010) também é feita a extensão dessa idéia para mais de duas hipóteses, considerado situações em que estamos interessados em sequências enumeráveis e não enumeráveis de hipóteses. Abaixo introduziremos uma classe de testes bastante simples que atende a essa propriedade, e também as propriedades de monotonicidade e invertibilidade, e que será importante no final desse capítulo.

Exemplo 2.5 (Classe de testes baseada em um estimador pontual - Silva (2014)). Seja $\delta: \chi \rightarrow \Theta$ um estimador pontual para o parâmetro $\theta$. A classe de testes $\mathcal{L}$ gerada por $\delta$ associa cada hipótese $A \in \sigma(\Theta)$ ao teste que aceita a hipótese se $\delta(x) \in A$ e rejeita caso contrário:

$$
\mathcal{L}(A)(x)= \begin{cases}0, & \text { se } \delta(x) \in A \\ 1, & \text { se } \delta(x) \notin A\end{cases}
$$

Essa classe de testes atende a propriedade de consonância da união, pois para $A, B \in \sigma(\Theta)$ temos algumas alternativas: se $\delta(x) \in A$ ou $\delta(x) \in B, \delta(x) \in A \cup B$ e, portanto, vale que $\mathcal{L}(A \cup B)=$ $\mathcal{L}(A) \mathcal{L}(B)$, pois $\mathcal{L}(A \cup B)=0$ e $\mathcal{L}(A)=0$ ou $\mathcal{L}(B)=0$. Se $\delta(x) \notin A \cup B$, então $\delta(x) \notin A$ e $\delta(x) \notin B$, de forma que $\mathcal{L}(A \cup B)=1, \mathcal{L}(A)=1$ e $\mathcal{L}(B)=1$. Então, também vale que $\mathcal{L}(A \cup B)=\mathcal{L}(A) \mathcal{L}(B)$.

\subsection{Consonância da Intersecção}

De forma similar a propriedade anterior, essa propriedade afirma que se aceitarmos duas ou mais hipóteses simultaneamente, devemos também aceitar a hipótese gerada pela intersecção dos conjuntos de distribuições especificados por todas elas.

Definição 2.6 (Consonância da intersecção finita - Izbicki (2010)). Uma classe de testes $\mathcal{L}$ atende a propriedade de consonância da intersecção finita se, $\forall A, B \in \sigma(\Theta), \mathcal{L}(A \cap B) \leq$ $\max \{\mathcal{L}(A), \mathcal{L}(B)\}$.

Izbicki (2010) também estende essa idéia para mais de duas hipóteses, considerando sequências de hipóteses enumeráveis e não enumeráveis. A classe de testes baseada em um estimador pontual (Exemplo 2.5) também atende essa propriedade, a seguir, trazemos um exemplo de teste onde essa propriedade não é atendida:

Exemplo 2.6 (Classe de testes baseada em um estimador por região - Lindley (1970)). Essa classe de testes foi adaptada de Lindley (1970), onde, para testar uma hipótese de um único elemento $\left(A=\left\{\theta_{0}\right\}\right)$, verifica-se se ele está presente em um intervalo de confiança para $\theta$. Seja $R: \chi \rightarrow\{A: A \subseteq \Theta\}=\mathcal{P}(\Theta)$ um estimador por região para o parâmetro $\theta$. A classe de testes $\mathcal{L}$ gerada por $R$ associa cada hipótese $A \in \sigma(\Theta)$ ao teste que rejeita a hipótese se $R(x) \cap A=\emptyset \mathrm{e}$ aceita caso contrário:

$$
\mathcal{L}(A)(x)= \begin{cases}1, & \text { se } R(x) \cap A=\emptyset \\ 0, & \text { caso contrário }\end{cases}
$$

Essa classe de testes não atende a propriedade de consonância da intersecção, pois é possível que para duas hipóteses $A$ e $B$ onde $A \cap B \neq \emptyset$, tenhamos uma estimativa por região $R(x)$ tal que $R(x) \cap A \neq \emptyset, R(x) \cap B \neq \emptyset \operatorname{mas} R(x) \cap(A \cap B)=\emptyset$. 


\subsection{Propriedades em Conjunto}

Considerando essas propriedades, faz sentido se perguntar se existem testes que apresentem todas essas características conjuntamente e que relações essas propriedades tem entre si. Em Izbicki (2010) foram definidas três desideratas, conjuntos de propriedades que classes de testes podem atender simultâneamente:

- Desiderata 1 (D1): Uma classe de testes $\mathcal{L}$ satisfaz a desiderata D1 se ela atende as propriedades de invertibilidade e monotonicidade.

- Desiderata 2 (D2): Uma classe de testes $\mathcal{L}$ satisfaz a desiderata D2 se ela atende as propriedades de invertibilidade, monotonicidade e consonância da união finita. Aqui, uma consequência das propriedades anteriores é que essa classe de testes atenderá a propriedade de consonância da intersecção finita.

- Desiderata 3 (D3): Uma classe de testes $\mathcal{L}$ satisfaz a desiderata D3 se ela atende as propriedades de invertibilidade, monotonicidade e consonância da união enumerável. Novamente, uma consequência das propriedades anteriores é que essa classe de testes atenderá a propriedade de consonância da intersecção enumerável.

No mesmo trabalho, um resultado importante foi obtido a respeito das classes de testes que satisfazem a desiderata mais restritiva, D3:

Resultado 2.1 (Izbicki (2010)). Suponha que $\Theta=\mathbb{R}^{n}$ e $\mathcal{B}(\Theta) \subseteq \sigma(\Theta)$. Então, uma classe $\mathcal{L}$ satisfaz a desiderata D3 se, e somente se, para cada $x \in \chi, \exists$ ! $\theta_{0}=\theta_{0}(x) \in \Theta$ tal que $\mathcal{L}(A)(x)=$ $0 \Longleftrightarrow \theta_{0} \in A, \forall A \in \sigma(\Theta)$. Isto é, vale D3 se, e somente se, $\forall A \in \sigma(\Theta)$ :

$$
\mathcal{L}(A)(x)= \begin{cases}0, & \text { se } \theta_{0}(x) \in A \\ 1, & \text { se } \theta_{0}(x) \notin A\end{cases}
$$

Ou seja, se $\mathcal{L}$ é uma classe de testes que atende a todas as propriedades citadas anteriormente, então ela liga cada hipótese possível $A$ à uma decisão de aceitar a hipótese se, e somente se, a função da amostra no ponto $x, \theta_{0}(x)$, uma estimativa pontual, for um dos elementos de $A$ (Exemplo 2.5).

Sob a ótica da teoria da decisão bayesiana, Silva (2014) discute quais restrições devem ser impostas às famílias de funções de perda para que as respectivas classes de testes de Bayes (Definição 2.3) sigam cada uma das propriedades discutidas, para qualquer distribuição a priori de $\theta$. As conclusões obtidas foram que, apesar de existirem famílias de funções de perda que geram classes de testes de Bayes satisfazendo à desiderata D1 para qualquer priori, não existe nenhuma família de funções de perda tal que, para qualquer priori, a classe de testes de Bayes gerada satisfaça a desiderata D2, considerando algumas suposições bastante razoáveis. Apesar disso, é apresentado um resultado sugerindo que satisfazer a desiderata D2 é possível, sob certas condições:

Resultado 2.2 (Silva (2014)). Sejam $\Theta$ e $\chi$ finitos, e seja $\sigma(\Theta)=\mathcal{P}(\Theta)$ o conjunto das partes de $\Theta$. Se uma classe de testes de hipóteses satisfaz a desiderata D2, então existem uma medida de probabilidade $\mathbb{P}: \sigma(\Theta \times \chi) \rightarrow[0,1]$ e uma familia de funçôes de perda $\left(L_{A}\right)_{A \in \sigma(\Theta)}$ satisfazendo $L(1, \theta) \geq L(0, \theta)$ para $\theta \in A$ e $L(1, \theta) \leq L(0, \theta)$ para $\theta \notin A$, tais que essa classe é classe de testes de Bayes (Definição 2.4) gerada por $\left(L_{A}\right)_{A \in \sigma(\Theta)}$ contra a $\mathbb{P}$-marginal de $\theta$. 
Ou seja, uma classe de testes que atende às propriedades que compõe a desiderata D2 é o teste de Bayes de algum agente decisor. Além disso, outro resultado complementa o resultado 2.1 de Izbicki (2010), citado acima, concluindo que o estimador que gera um teste que satisfaz a desiderata D2 é de Bayes contra alguma perda $L$ e priori $\pi$, sob algumas restrições.

Resultado 2.3 (Silva (2014)). Sejam $\Theta$ e $\chi$ e seja $\left(\phi_{A}\right)_{A \in \sigma(\Theta)}$ a classe de testes de hipótese gerada por um estimador pontual $\delta: \chi \rightarrow \Theta$ (Exemplo 2.5) e que portanto, satisfaz a desiderata D2. Suponha que a verossimilhança $V_{x}(\delta(x))$ é positiva para todo $x \in \chi$ :

(a) Se existem $\pi: \sigma(\Theta) \rightarrow[0,1]$, medida de probabilidade a priori para $\theta$ com $\pi(\delta(x))$ positivo para todo $x \in \chi$ e $\bar{L}: \Theta \times \Theta \rightarrow \mathbb{R}_{+}$, com $\bar{L}(\theta, \theta)=0$ e $\bar{L}(d, \theta)$ positivo para $d \neq \theta$, tais que $\delta$ é estimador de Bayes gerado por $\bar{L}$ contra $\pi$, então existe $\left(L_{A}\right)_{A \in \sigma(\Theta)}$ com $L_{A}:\{0,1\} \times \Theta \times \chi \rightarrow \mathbb{R}_{+}$para cada $A \in \sigma(\theta)$, tal que $\left(\phi_{A}\right)_{A \in \sigma(\Theta)}$ é classe de testes de Bayes gerada por $\left(L_{A}\right)_{A \in \sigma(\Theta)}$ contra $\pi$.

(b) Se existem $\pi: \sigma(\Theta) \rightarrow[0,1]$, medida de probabilidade a priori para $\theta$ com $\pi(\delta(x))$ positivo para todo $x \in \chi$ e existe $\left(L_{A}\right)_{A \in \sigma(\Theta)}$ com $L_{A}:\{0,1\} \times \Theta \rightarrow \mathbb{R}_{+}$satisfazendo $L(0, \theta)<L(1, \theta)$ para $\theta \in A$ e $L(0, \theta)>L(1, \theta)$ para $\theta \notin A$ para cada $A \in \sigma(\Theta)$ tais que $\left(\phi_{A}\right)_{A \in \sigma(\Theta)}$ é classe de testes de Bayes gerada por $\left(L_{A}\right)_{A \in \sigma(\Theta)}$ contra $\pi$, então existe $\bar{L}: \Theta \times \Theta \rightarrow \mathbb{R}_{+}$, com $\bar{L}(\theta, \theta)=0$ e $\bar{L}(d, \theta)$ positivo para $d \neq \theta$, tais que $\delta$ é estimador de Bayes gerado por $\bar{L}$ contra $\pi$.

Todos esses resultados mostram que conciliar a consistência lógica total, representada pelas desideratas D2 e D3, com os testes de hipótese usuais, nos limitam a regras de decisão muito simples, baseadas apenas nas estimativas pontuais fornecidas pela amostra. 


\section{Capítulo 3}

\section{Testes de Hipóteses Agnósticos}

Dadas as limitações dos testes hipóteses usuais vistas no capítulo anterior ao procurar manter coerência lógica em testes simultâneos, uma alternativa é expandir o espaço de possíveis decisões, originalmente apenas aceitar ou rejeitar uma hipótese, adicionando uma terceira alternativa: suspender a escolha por uma das alternativas anteriores e permanecer agnóstico sobre a hipótese quando a evidência fornecida pela amostra for insuficiente para escolher uma das duas opções.

Essa estrutura de problema de tomada de decisão já foi discutida em Berg (2004), onde o autor propõe as opções de rejeitar a hipótese nula, rejeitar a hipótese alternativa ou nenhuma das duas, efetivamente não tomando nenhuma decisão e permitindo o controle dos erros de tipo I e II pelas regiões de rejeição.

Dentro do contexto de classificação binária em aprendizado de máquina, essa idéia também aparece como classificação seletiva em El-Yaniv e Wiener (2010), ou classificação com opção de rejeição em Chow (1970), onde modelos preditivos tem a opção de não classificar observações que tem pouca confiança na sua previsão, melhorando as métricas de performance mas diminuindo os casos em que ele faz previsões (a cobertura dos modelos).

Recentemente, alguns trabalhos tem resgatado essas idéias para o contexto de testes de hipóteses: Esteves et al. (2016) define classes de testes de hipóteses agnósticos e adapta as propriedades discutidas no capítulo anterior, buscando coerência lógica nesse contexto e investigando também, sob a ótica da teoria de decisão bayesiana, restrições para que funções de perdas gerem testes de Bayes atendendo cada uma das propriedades individualmente. Coscrato et al. (2018) procura adaptar as idéias de teste uniformemente mais poderosos aos testes de hipóteses agnósticos.

\subsection{Definições Iniciais}

Aqui vamos manter a maioria das definições do capítulo anterior, mas agora, para cada amostra observada $x$, queremos afirmar se ela é compatível com o conjunto de distribuições $\left\{\mathbb{P}_{\theta_{0}}: \theta_{0} \in \Theta_{0}\right\}$ induzido pelo conjunto de parâmetros $\Theta_{0}$ (nossa hipótese), se ela é compatível com a hipótese alternativa, que é o conjunto de parâmetros $\Theta_{1}$ que induz a família de distribuições $\left\{\mathbb{P}_{\theta_{1}}: \theta_{1} \in \Theta_{1}\right\}$ ou se não podemos razoavelmente afirmar nenhuma das duas alternativas, permanecendo agnóstico.

Para isso, utilizaremos uma função de teste $\phi: \chi \rightarrow\left\{0, \frac{1}{2}, 1\right\}$ que associa a cada amostra possível $x$ a decisão de rejeitar a hipótese (representada por $\phi(x)=1$ ) quando os dados trouxerem evidência favorável a $H_{1}: \theta \in \Theta_{1}$, a decisão de aceitar a hipótese (representada por $\phi(x)=0$ ) quando trouxerem evidência favorável a $H_{0}: \theta \in \Theta_{0}$, ou a decisão de permanecer agnóstico (representada 
por $\left.\phi(x)=\frac{1}{2}\right)$.

De forma análoga aos testes de hipóteses usuais, utilizaremos o conceito de classe de testes de hipóteses agnósticos para associar, a cada hipótese possível, um teste de hipótese agnóstico apropriado.

Definição 3.1 (Classe de testes de hipóteses agnósticos - Esteves et al. (2016)). Uma classe de testes de hipóteses agnósticos pode ser definida como uma função $\mathcal{L}: \sigma(\Theta) \rightarrow \Phi$, onde $\sigma(\Theta)$ é uma $\sigma$-álgebra do espaço paramétrico e $\Phi=\left\{\phi: \chi \rightarrow\left\{0, \frac{1}{2}, 1\right\}\right\}$ é o conjunto de todas as funçôes de testes agnósticos possíveis. Dessa forma, $\mathcal{L}$ associa a cada possível hipótese uma função de teste para a amostra. Podemos também representar a classe por meio das funçôes de testes indexadas pelos conjuntos de $\sigma(\Theta),\left(\phi_{A}\right)_{A \in \sigma(\Theta)}$

Exemplo 3.1 (Classe de testes de hipóteses agnósticos baseada em probabilidades a posteriori Esteves et al. (2016)). Sejam $\Theta=\mathbb{R}^{n}, \sigma(\Theta)=\mathcal{B}(\Theta)$ os borelianos de $\mathbb{R}^{n}$ e $\pi$ uma distribuição de probabilidade em $\sigma(\Theta)$ fixada. Para $c_{1}, c_{2} \in(0,1), c_{1} \geq c_{2}, A \in \sigma(\Theta)$, definimos $\mathcal{L}(A): \chi \rightarrow\left\{0, \frac{1}{2}, 1\right\}$ por:

$$
\mathcal{L}(A)(x)= \begin{cases}0, & \text { se } \mathbb{P}(A \mid x)>c_{1} \\ \frac{1}{2}, & \text { se } c_{1} \geq \mathbb{P}(A \mid x) \geq c_{2} \\ 1, & \text { se } c_{2}>\mathbb{P}(A \mid x)\end{cases}
$$

onde $\mathbb{P}(. \mid x)$ é a distribuição a posteriori de $\theta \mid x$.

Esse teste basicamente nos permite decidir entre a aceitação ou rejeição de uma hipótese dependendo de quão provável ela é em vista dos dados: aceitamos a hipótese nos casos em que ela tem probabilidade a posteriori alta, rejeitamos a hipótese nos casos em que a sua probabilidade a posteriori é baixa e permanecemos agnósticos nos casos onde temos valores de probabilidade a posterori intermediários para nossa hipótese.

No exemplo acima, $c_{1}-c_{2}$, assumindo valores em $[0,1]$, representa o quanto o indivíduo se permite permanecer agnóstico. Nos casos em que a diferença $c_{1}-c_{2}$ é grande, o indivíduo é muito resistente a tomar uma decisão sem ter grande convicção sobre a hipótese em consideração: em situações limite, nas quais essa diferença se aproxima de 1, praticamente só aceitamos hipóteses com probabilidade 1 e rejeitamos hipóteses com probabilidade 0. Por outro lado, quando a diferença $c_{1}-c_{2}$ é pequena, o individuo é mais propenso a tomar uma decisão, mesmo em situações que parecem ser mais ambíguas, até o limite onde essa diferença se aproxima de 0 e temos um teste de hipótese bayesiano usual (com duas alternativas) para $A$, sem a decisão de permanecer agnóstico.

Nos mesmos moldes do exemplo anterior, poderíamos substituir a probabilidade a posteriori de uma hipótese por qualquer outra medida de suporte (Schervish (1996)) à hipótese de interesse baseada em $x$, como razão de verossimilhanças (Lindgren (1993)), fatores de Bayes (Robert (2007)) e o valor de evidência de Pereira-Stern (Pereira e Stern (1999)). Outros exemplos de medidas de suporte possíveis podem ser encontrados em Fossaluza et al. (2017).

Exemplo 3.2 (Classe de testes de hipóteses agnósticos baseada em uma estatística de suporte Esteves et al. (2016)). Nas condições do exemplo anterior, seja, para qualquer hipótese $A \in \sigma(\Theta), s_{A}: \chi \rightarrow \mathbb{R}$ uma estatística. Para $c_{1}, c_{2} \in \mathbb{R}, c_{1} \geq c_{2}, A \in \sigma(\Theta)$, podemos definir 
$\mathcal{L}(A): \chi \rightarrow\left\{0, \frac{1}{2}, 1\right\}$ como:

$$
\mathcal{L}(A)(x)= \begin{cases}0, & \text { se } s_{A}(x)>c_{1} \\ \frac{1}{2}, & \text { se } c_{1} \geq s_{A}(x) \geq c_{2} \\ 1, & \text { se } c_{2}>s_{A}(x)\end{cases}
$$

No exemplo 3.1, se considerarmos uma família de funções de perda que para cada hipótese possível $A \in \sigma(\Theta)$, liga cada decisão (aceitar, rejeitar ou permanecer agnóstico) e estado real da natureza (se a hipótese é verdadeira ou não) a um custo, podemos ter testes de Bayes com $c_{1}$ e $c_{2}$ diferentes para cada hipótese. Em geral, podemos construir testes agnósticos de Bayes simultâneos através da teoria da decisão, conforme a seguinte definição.

Definição 3.2 (Classe de testes de hipóteses agnósticos gerados por uma família de funções de perda - Esteves et al. (2016)). Seja $\pi$ uma distribuição de probabilidade em $\sigma(\Theta)$, $\left(L_{A}\right)_{A \in \sigma(\Theta)}$ uma familia de funçôes de perda $L_{A}: \Theta \times\left\{0, \frac{1}{2}, 1\right\} \rightarrow \mathbb{R}$ que associa à hipótese $A$ os custos de tomada de cada decisão. Temos que $\mathcal{L}$ é uma classe de testes de hipóteses agnósticos gerados por $\left(L_{A}\right)_{A \in \sigma(\Theta)}$ se para toda hipótese $A \in \sigma(\Theta), \mathcal{L}(A)$ é uma regra de decisão de Bayes segundo $L_{A}$ contra $\pi$ no espaço de decisões $\left\{0, \frac{1}{2}, 1\right\}$.

É importante ressaltar que, na definição acima, não impomos nenhuma restrição sobre $\left(L_{A}\right)_{A \in \sigma(\Theta)}$, porém, nem toda família de funções de perda gera regras de decisão que fazem sentido como testes de hipóteses. Por exemplo, é possível que $\left(L_{A}\right)_{A \in \sigma(\Theta)}$ seja tal que, $L_{A}(\theta, 0)>L_{A}(\theta, 1)$ quando $\theta \in A$, nesse caso, uma decisão correta (não rejeitar $A$ ) teria uma penalidade maior do que uma decisão incorreta (rejeitar $A$ ) quando $\theta \in A$.

Nos testes de hipóteses usuais, uma das restrições sobre $L$ é justamente que $L_{A}(\theta, 0)<L_{A}(\theta, 1)$ quando $\theta \in A$ e $L_{A}(\theta, 0)>L_{A}(\theta, 1)$ quando $\theta \notin A$ (Schervish (1995)). Para os testes de hipóteses agnósticos isso será examinado com cuidado na seção 3.4.

\subsection{Propriedades Lógicas nos Testes de Hipóteses Agnósticos}

A inclusão de uma nova resposta possível do teste requer algumas alterações na definição das propriedades trazidas no capítulo anterior. Nessa seção, revisitaremos essas propriedades, fazendo as adaptações necessárias para os testes de hipóteses agnósticos.

\subsubsection{Monotonicidade}

Definição 3.3 (Monotonicidade - Esteves et al. (2016)). Uma classe de testes de hipóteses agnósticos $\mathcal{L}$ atende a propriedade de monotonicidade (é monotônica) se, $\forall A, B \in \sigma(\Theta), A \subseteq B \Rightarrow$ $\mathcal{L}(A) \geq \mathcal{L}(B)$.

Ou seja, como nos testes de hipóteses usuais, para uma amostra $x$ observada, se uma hipótese $A$ for aceita $(\mathcal{L}(A)(x)=0)$, todas as hipóteses que contém $A$ deverão ser aceitas (isto é, $\mathcal{L}(B)(x)=0$, $\forall B \supseteq A)$. Agora, adicionalmente, se ficarmos agnósticos a respeito da hipótese $A\left(\mathcal{L}(A)(x)=\frac{1}{2}\right)$, não podemos rejeitar hipóteses que contém $A$ (isto é, devemos ter $\mathcal{L}(B)(x)=0$ ou $\mathcal{L}(B)(x)=\frac{1}{2}, \forall$ $B \supseteq A)$. Os testes baseados em probabilidades a posteriori são um exemplo de situação onde essa propriedade é atendida: 
Exemplo 3.3 (Classe de testes de hipóteses agnósticos baseada em probabilidades a posteriori Esteves et al. (2016)). Consideremos novamente o teste do exemplo 3.1, onde aceitamos uma hipótese se a probabilidade a posteriori da hipótese $\mathbb{P}(\theta \in A \mid x)$ for maior que um valor $c_{1}$, ficamos agnósticos se esse valor estiver entre $c_{2}$ e $c_{1}$ e rejeitamos se estiver abaixo de $c_{2}$. Como $A \subseteq B$, temos que $\mathbb{P}(\theta \in A \mid x) \leq \mathbb{P}(\theta \in B \mid x)$, e portanto, essa classe de testes é monotônica, pois sempre teremos $\mathcal{L}(A)(x) \geq \mathcal{L}(B)(x)$.

É de se esperar que essa propriedade não valha para todas as classes de testes, mesmo agnósticas. Esteves et al. (2016) apresenta condições para que outras medidas de suporte possam ser utilizadas no lugar da probabilidade a posteriori (Exemplo 3.2) e mantenham a monotonicidade. Um exemplo de estatística que é frequentemente usada e que nem sempre atende tal propriedade é o fator de Bayes. Essa estatística é utilizada para seleção de modelos, mas também pode ser utilizada para testes de hipóteses (Robert (2007)).

Exemplo 3.4 (Classe de testes de hipóteses agnósticos baseada no fator de Bayes Lavine e Schervish (1999)). Considere o experimento onde jogamos uma moeda quatro vezes e contamos o número de ocorrências em que resultado foi cara. Suponha que essa moeda pode ser com duas caras, duas coroas ou uma cara e uma coroa. Seja $\theta$ o número de caras que compõe a moeda. Temos então que $\Theta=\{0,1,2\}, X \mid \theta \sim \operatorname{Bin}\left(4, \frac{\theta}{2}\right)$ e $\chi=\{0,1, \ldots, 4\}$. Seja $\sigma(\Theta)=\mathcal{P}(\Theta)$ o conjunto das partes de $\Theta$ e a distribuição a priori para $\theta$ como $\mathbb{P}(\theta=0)=0.01, \mathbb{P}(\theta=1)=0.98 \mathrm{e}$ $\mathbb{P}(\theta=2)=0.01$. Para uma hipótese $A \in \sigma(\Theta)$, definimos o fator de Bayes como:

$$
\mathrm{FB}_{A}(x)=\frac{\mathbb{P}(\theta \in A \mid x)}{\mathbb{P}(\theta \notin A \mid x)} / \frac{\mathbb{P}(\theta \in A)}{\mathbb{P}(\theta \notin A)}
$$

Então, para $c_{1}, c_{2} \in \mathbb{R}, c_{1} \geq c_{2}$, definimos $\mathcal{L}(A): \chi \rightarrow\left\{0, \frac{1}{2}, 1\right\}$ como:

$$
\mathcal{L}(A)(x)= \begin{cases}0, & \text { se } \mathrm{FB}_{A}(x)>c_{1} \\ \frac{1}{2}, & \text { se } c_{1} \geq \mathrm{FB}_{A}(x) \geq c_{2} \\ 1, & \text { se } c_{2}>\mathrm{FB}_{A}(x)\end{cases}
$$

Se observarmos quatro caras $(x=4)$, para as hipóteses $A=\{1\}$ e $B=\{0,1\}$ temos que:

$$
\mathrm{FB}_{A}(x)=\frac{1}{8}=0.125 \text { e } \mathrm{FB}_{B}(x)=\frac{98}{1584} \approx 0.0619
$$

Então, dependendo da escolha de $c_{1}$ e $c_{2}$, nossa conclusão do teste pode não ser compatível com a propriedade de monotonicidade, pois temos que $A \subset B$ e a estatística escolhida para representar o suporte as hipóteses é menor para $B$ do que para $A$. Por exemplo, se $c_{2}=0.1$ e $c_{2}=0.5$, o resultado do teste seria permanecer agnóstico sobre a hipótese da moeda ter uma cara mas rejeitar a hipótese de que a moeda tem uma cara ou nenhuma cara, o que não parece razoável.

\subsubsection{Invertibilidade}

Definição 3.4 (Invertibilidade - Esteves et al. (2016)). Uma classe de testes de hipóteses agnósticos $\mathcal{L}$ atende a propriedade de invertibilidade (é invertível) se, $\forall A \in \sigma(\Theta), \mathcal{L}(A)=1-\mathcal{L}\left(A^{c}\right)$.

Ou seja, como nos testes de hipóteses usuais, para qualquer hipótese $A$, sua rejeição implica na 
aceitação da hipótese alternativa $A^{c}$ quando testada e vice-versa. Adicionalmente, se o resultado do teste é permanecer agnóstico sobre $A$, devemos necessariamente permanecer agnósticos quando testamos a hipótese $A^{c}$. No exemplo 3.1, dependendo da escolha de $c_{1}$ e $c_{2}$ isso pode não ocorrer (mais especificamente, se $c_{2} \neq 1-c_{1}$, como visto em Esteves et al. (2016)). A seguir, trazemos um exemplo de teste que atende a essa propriedade e será importante mais a frente nesse capítulo.

Exemplo 3.5 (Classe de testes de hipóteses agnósticos baseada em estimadores por região - Esteves et al. (2016)). Seja $R: \chi \rightarrow \mathcal{P}(\Theta)$ um estimador por região de $\theta$. Para uma hipótese $A \in \sigma(\Theta)$, podemos definir $\mathcal{L}(A): \chi \rightarrow\left\{0, \frac{1}{2}, 1\right\}$ como:

$$
\mathcal{L}(A)(x)= \begin{cases}0, & \text { se } R(x) \subseteq A \\ 1, & \text { se } R(x) \subseteq A^{c} \\ \frac{1}{2}, & \text { caso contrário }\end{cases}
$$

Ou seja, nessa classe de testes:

- Aceitamos a hipótese $A$ quando a estimativa por região $R(x)$ está contida em $A$. Nesse caso, os dados parecem ser compatíveis com a hipótese $A$.

- Rejeitamos a hipótese $A$ quando $R(x)$ não tem nenhum elemento em comum com $A$. Aqui, os dados parecem ser incompatíveis com a hipótese $A$ e, por argumento similar ao caso anterior, compatíveis com a hipótese alternativa $A^{c}$.

- Permanecemos agnósticos a respeito da hipótese quando $R(x)$ tem elementos em $A$ e $A^{c}$, pois os dados parecem não sustentar uma única hipótese como nas situações anteriores, não fornecendo evidências suficientes para a decisão entre uma delas.

Essa classe de testes é invertível, pois, para os casos em que $\mathcal{L}(A)(x)=0, R(x) \subseteq A$ e, portanto, $\mathcal{L}\left(A^{c}\right)(x)=1$; para os casos em que $\mathcal{L}(A)(x)=1, R(x) \subseteq A^{c}$ e, portanto, $\mathcal{L}\left(A^{c}\right)(x)=0$; finalmente, se $\mathcal{L}(A)(x)=\frac{1}{2}, R(x)$ não está contido em $A$ nem em $B$ e, portanto, $\mathcal{L}\left(A^{c}\right)(x)=\frac{1}{2}$.

\subsubsection{Consonância da União}

Nessa propriedade, a inclusão da possibilidade de permanecer agnóstico com relação a uma hipótese requer que façamos uma distinção entre possíveis formas de consonância da união.

Definição 3.5 (Consonância fraca da união - Esteves et al. (2016)). Uma classe de testes de hipóteses agnósticos $\mathcal{L}$ atende a propriedade de consonância fraca da união se, $\forall A, B \in \sigma(\Theta)$ $\mathcal{L}(A)(x)=1$ e $\mathcal{L}(B)(x)=1 \Rightarrow \mathcal{L}(A \cup B)(x) \neq 0$.

Ou seja, se rejeitarmos duas hipóteses definidas por $A$ e $B$ quando testadas isoladamente, não devemos aceitar a hipótese gerada pela união das duas. Aqui podemos até permanecer agnósticos quando os dados se mostrarem incompatíveis com as hipóteses $A$ e $B$, quando avaliadas separadamente. Uma alternativa mais forte dessa propriedade não se limita a pares, considerando qualquer sequência possívelmente não enumerável de hipóteses, e exige que rejeitemos a hipótese formada pela união delas quando as rejeitamos individualmente. 
Definição 3.6 (Consonância forte da união - Esteves et al. (2016)). Uma classe de testes de hipóteses agnósticos $\mathcal{L}$ atende a propriedade de consonância forte da união se, para todo conjunto $I$ de indices arbitrário e $\left\{A_{i}\right\}_{i \in I}$, com $A_{i} \in \sigma(\Theta) \forall i \in I$ e tal que $\cup_{i \in I} A_{i} \in \sigma(\Theta)$, $\min \left\{\mathcal{L}\left(A_{i}\right)(x)\right\}_{i \in I}=1 \Rightarrow \mathcal{L}\left(\cup_{i \in I} A_{i}\right)(x)=1$.

A classe de testes de hipóteses agnósticos baseada em estimadores por região (Exemplo 3.5) atende a propriedade de consonância forte da união, e, consequentemente, também atende a propriedade de consonância fraca da união. O exemplo a seguir atende a propriedade de consonância fraca da união mas não a consonância forte da união:

Exemplo 3.6. Seja $\Theta=\mathbb{N}$, o conjunto dos números naturais (considerando o número zero), e consideremos que, para uma amostra observada $x, \theta \mid X=x \sim \operatorname{Geom}\left(\frac{1}{5}\right)$. Se considerarmos a classe de testes de hipóteses agnósticos baseada em probabilidades a posteriori do exemplo $\mathbf{3 . 1} \operatorname{com} c_{1}=\frac{3}{4}$ e $c_{2}=\frac{1}{4}$, temos que:

$$
\mathcal{L}(A)(x)= \begin{cases}0, & \text { se } \mathbb{P}(A \mid x)>\frac{3}{4} \\ \frac{1}{2}, & \text { se } \frac{3}{4} \geq \mathbb{P}(A \mid x) \geq \frac{1}{4} \\ 1, & \text { se } \frac{1}{4}>\mathbb{P}(A \mid x)\end{cases}
$$

Verificaremos ainda nesse capítulo que essa classe de testes atende a propriedade da consonância fraca da união. Se considerarmos a sequência de hipóteses $A_{i}=\{i\}$, temos que $\mathbb{P}\left(A_{i} \mid x\right)<\frac{1}{4}$ e $\mathcal{L}\left(A_{i}\right)(x)=1, \forall i \in \mathbb{N}$. Por outro lado, $\mathbb{P}\left(\cup_{i \in \mathbb{N}} A_{i} \mid x\right)=\mathbb{P}(\Theta \mid x)=1$ e então $\mathcal{L}\left(\cup_{i \in \mathbb{N}} A_{i}\right)(x)=0$. Portanto, essa classe de testes não atende a propriedade de consonância forte da união.

\subsubsection{Consonância da Intersecção}

De forma análoga a consonância da união, precisamos distinguir a consonância da intersecção entre fraca e forte.

Definição 3.7 (Consonância fraca da intersecção - Esteves et al. (2016)). Uma classe de testes de hipóteses agnósticos $\mathcal{L}$ atende a propriedade de consonância fraca da intersecção se, $\forall A$, $B \in \sigma(\Theta), \mathcal{L}(A)(x)=0$ e $\mathcal{L}(B)(x)=0 \Rightarrow \mathcal{L}(A \cap B)(x) \neq 1$.

Ou seja, se aceitarmos duas hipóteses definidas por $A$ e $B$, não devemos rejeitar a hipótese gerada pela intersecção dos dois conjuntos de distribuições $A \cap B$, mas podemos permanecer agnósticos. Aqui, novamente, uma alternativa mais forte dessa propriedade não se restringe apenas a quando analisamos as hipóteses duas a duas e diz que quando aceitamos todas as hipóteses de uma sequência qualquer, também devemos aceitar a hipótese formada pela intersecção delas.

Definição 3.8 (Consonância forte da intersecção - Esteves et al. (2016)). Uma classe de testes de hipóteses agnósticos $\mathcal{L}$ atende a propriedade de consonância forte da intersecção se, para todo conjunto $I$ de indices arbitrário $e\left\{A_{i}\right\}_{i \in I}$, com $A_{i} \in \sigma(\Theta) \forall i \in I$ e tal que $\cap_{i \in I} A_{i} \in \sigma(\Theta)$ $\max \left\{\mathcal{L}\left(A_{i}\right)(x)\right\}_{i \in I}=0 \Rightarrow \mathcal{L}\left(\cap_{i \in I} A_{i}\right)(x)=0$.

A classe de testes de hipóteses agnósticos baseada em estimadores por região (Exemplo 3.5) atende também a propriedade de consonância forte da intersecção. A seguir trazemos um exemplo de classe de testes que atende a propriedade de consonância fraca da intersecção mas não a consonância forte da intersecção: 
Exemplo 3.7. De forma similar ao exemplo 3.6, seja $\Theta=\mathbb{N}$, o conjunto dos números naturais (considerando o número zero), e consideremos que, para uma amostra osbervada $x, \theta \mid X=x \sim$ $\operatorname{Geom}\left(\frac{4}{5}\right)$. Se considerarmos a classe de testes de hipóteses agnósticos baseada em probabilidades a posteriori do exemplo $\mathbf{3 . 1}$ com $c_{1}=\frac{4}{5}$ e $c_{2}=\frac{1}{5}$, temos que:

$$
\mathcal{L}(A)(x)= \begin{cases}0, & \text { se } \mathbb{P}(A \mid x)>\frac{4}{5} \\ \frac{1}{2}, & \text { se } \frac{4}{5} \geq \mathbb{P}(A \mid x) \geq \frac{1}{5} \\ 1, & \text { se } \frac{1}{5}>\mathbb{P}(A \mid x)\end{cases}
$$

Verificaremos ainda nesse capítulo que essa classe de testes atende a propriedade da consonância fraca da intersecção. Se considerarmos a sequência de hipóteses $A_{i}=\{0\} \cup\{k: k>i, k \in \mathbb{N}\}$, temos que $\mathbb{P}\left(A_{i} \mid x\right)>\frac{4}{5}$ e $\mathcal{L}\left(A_{i}\right)(x)=0, \forall i \in \mathbb{N}$. Por outro lado, $\mathbb{P}\left(\cap_{i \in \mathbb{N}} A_{i} \mid x\right)=\mathbb{P}(\theta=0 \mid x)=\frac{4}{5}$ e então $\mathcal{L}\left(\cap_{i \in \mathbb{N}} A_{i}\right)(x)=\frac{1}{2}$. Portanto, essa classe de testes não atende a propriedade de consonância forte da intersecção.

Na próxima seção trazemos os tipos de consistência lógica que resultam da exigência em conjunto das propriedades revistas acima, para testes de hipóteses agnósticos.

\subsection{Testes de Hipóteses Agnósticos Consistentes}

De forma análoga aos testes de hipóteses usuais, podemos investigar as classes de testes de hipóteses agnósticos com relação às propriedades definidas anteriormente em conjunto. Para isso, Esteves et al. (2016) define também três desideratas categorizadas em dois tipos, que caracterizam classes de testes de hipóteses agnósticos fortemente consistentes e fracamente consistentes.

\subsubsection{Consistência Fraca}

Definição 3.9 (Classe de testes de hipóteses agnósticos fracamente consistente - Esteves et al. (2016)). Uma classe de testes de hipóteses agnósticos $\mathcal{L}$ é fracamente consistente se ela é monotônica (Definição 3.3), invertivel (Definição 3.4), atende a propriedade de consonância fraca da união (Definição 3.5) e a propriedade de consonância fraca da intersecção (Definiçãa 3.7).

Considerando as propriedades vistas na seção anterior, esse parece ser o conjunto de propriedades menos restritivas que uma classe de testes pode atender, que garante que, ao testarmos duas hipóteses simultâneamente, não obtenhamos contradições lógicas diretas. Abaixo, voltamos ao exemplo de teste baseado na probabilidade a posteriori da hipótese e trazemos as condições para que ele seja fracamente consistente:

Exemplo 3.8 (Classe de testes de hipóteses agnósticos baseada em probabilidades a posteriori - Esteves et al. (2016)). Retomando o exemplo 3.1, consideramos a seguinte classe de testes:

$$
\mathcal{L}(A)(x)= \begin{cases}0, & \text { se } \mathbb{P}(A \mid x)>c_{1} \\ \frac{1}{2}, & \text { se } c_{1} \geq \mathbb{P}(A \mid x) \geq c_{2}, \quad c_{1}, c_{2} \in[0,1] \\ 1, & \text { se } c_{2}>\mathbb{P}(A \mid x)\end{cases}
$$


Vamos avaliar essa classe de testes com respeito às propriedades que caracterizam consistência fraca: Monotonicidade: Vimos no exemplo $\mathbf{3 . 3}$ que ela é monotônica, pois, para quaisquer hipóteses $A$ e $B, A \subseteq B \Rightarrow \mathbb{P}(A \mid x) \leq \mathbb{P}(B \mid x)$.

Invertibilidade: Precisamos verificar que $\mathcal{L}(A)=1-\mathcal{L}\left(A^{c}\right)$, se $c_{2}=1-c_{1}$ :

$$
\begin{gathered}
\mathcal{L}(A)(x)=0 \Longleftrightarrow \mathbb{P}(A \mid x)>c_{1} \Longleftrightarrow 1-\mathbb{P}(A \mid x)<1-c_{1} \Longleftrightarrow \mathbb{P}\left(A^{c} \mid x\right)<c_{2} \Longleftrightarrow \mathcal{L}\left(A^{c}\right)(x)=1 \\
\mathcal{L}(A)(x)=1 \Longleftrightarrow \mathbb{P}(A \mid x)<c_{2} \Longleftrightarrow 1-\mathbb{P}(A \mid x)>1-c_{2} \Longleftrightarrow \mathbb{P}\left(A^{c} \mid x\right)>c_{1} \Longleftrightarrow \mathcal{L}\left(A^{c}\right)(x)=0 \\
\mathcal{L}(A)(x)=\frac{1}{2} \Longleftrightarrow c_{1} \geq \mathbb{P}(A \mid x) \geq c_{2} \Longleftrightarrow 1-c_{2} \geq 1-\mathbb{P}(A \mid x) \geq 1-c_{1} \Longleftrightarrow \\
\Longleftrightarrow c_{1} \geq \mathbb{P}\left(A^{c} \mid x\right) \geq c_{2} \Longleftrightarrow \mathcal{L}\left(A^{c}\right)(x)=\frac{1}{2}
\end{gathered}
$$

Assim, essa classe de testes é invertível se $c_{2}=1-c_{1}$

Consonância fraca da união: Consideremos $c_{1} \geq 2 c_{2}$, temos que:

$$
\mathbb{P}(A \cup B \mid x) \leq \mathbb{P}(A \mid x)+\mathbb{P}(B \mid x)
$$

Se rejeitarmos $A$ e $B$ individualmente, $\mathbb{P}(A \mid x)<c_{2}$ e $\mathbb{P}(B \mid x)<c_{2}$. Assim,

$$
\mathbb{P}(A \mid x)+\mathbb{P}(B \mid x)<2 c_{2}
$$

Então, $A \cup B$ nunca poderá ser aceita se $A$ e $B$ forem rejeitadas, pois $\mathbb{P}(A \cup B \mid x) \leq \mathbb{P}(A \mid x)+$ $\mathbb{P}(B \mid x)<2 c_{2} \leq c_{1}$, logo, $\mathcal{L}(A \cup B)(x) \neq 0$.

Consonância fraca da intersecção: Consideremos $c_{2} \leq 2 c_{1}-1$, temos que:

$$
\begin{gathered}
\mathbb{P}(A \cup B \mid x)=\mathbb{P}(A \mid x)+\mathbb{P}(B \mid x)-\mathbb{P}(A \cap B \mid x) \leq 1 \Rightarrow \\
\Rightarrow \mathbb{P}(A \cap B \mid x) \geq \mathbb{P}(A \mid x)+\mathbb{P}(B \mid x)-1
\end{gathered}
$$

Se aceitarmos $A$ e $B$ individualmente, $\mathbb{P}(A \mid x)>c_{1}$ e $\mathbb{P}(B \mid x)>c_{1}$. Assim,

$$
\mathbb{P}(A \cap B \mid x) \geq \mathbb{P}(A \mid x)+\mathbb{P}(B \mid x)-1>2 c_{1}-1
$$

Então, $A \cap B$ nunca poderá ser rejeitada se $A$ e $B$ forem aceitas.

Finalmente, a junção dessas restrições resulta que se $c_{1}>\frac{2}{3}$ e $c_{2}=1-c_{1}$, essa classe de testes será fracamente consistente.

Como visto no exemplo anterior, é possível obter testes baseados em probabilidades a posteriori de forma que as conclusões sejam fracamente consistentes, ao menos considerando duas hipóteses simultâneamente. Esteves et al. (2016) vai além e expande essa idéia para $n$ hipóteses, com a principal alteração sendo nas propriedades de consonância fraca para comportar o número de hipóteses apropriado. No entanto, para impor consistência lógica à tomada de decisão considerando um número qualquer $n$ de hipóteses, as restrições que devem ser impostas a $c_{1}$ e $c_{2}$ resultam que os testes só serão logicamente consistentes (fracamente) se o resultado for permanecer agnóstico para qualquer hipótese com probabilidade a posteriori em $(0,1)$. A seguir, trazemos o último tipo de consistência, mais restritivo. 


\subsubsection{Consistência Forte}

Definição 3.10 (Classe de testes de hipóteses fortemente consistente - Esteves et al. (2016)). Uma classe de testes de hipóteses agnósticos $\mathcal{L}$ é fortemente consistente se ela é monotônica (Definição 3.3), invertivel (Definição 3.4), atende a propriedade de consonância forte com a união (Definiçãa 3.6) e a propriedade de consonância forte com a intersecção (Definição $3.8)$.

Esse conjunto de propriedades garante que para qualquer combinação possível de hipóteses testadas, não incorreremos em inconsistências lógicas, considerando todas as propriedades vistas anteriormente. Agora, revisitaremos o teste baseado em estimadores por região, mostrando que ele é fortemente consistente.

Exemplo 3.9 (Classe de testes de hipóteses agnósticos baseada em estimadores por região - Esteves et al. (2016)). Retomando o exemplo 3.5, consideramos a seguinte classe de testes:

$$
\mathcal{L}(A)(x)= \begin{cases}0, & \text { se } R(x) \subseteq A \\ 1, & \text { se } R(x) \subseteq A^{c} \\ \frac{1}{2}, & \text { caso contrário }\end{cases}
$$

Vamos avaliar essa classe de testes com respeito às propriedades que caracterizam a consistência forte:

Monotonicidade: Considere $A \subseteq B$, se aceitamos $A$ então $R(x) \subseteq A$. Segue que $R(x) \subseteq B$ e, portanto, também aceitamos B. Da mesma forma, se ficarmos agnósticos sobre $A, R(x) \cap A \neq \varnothing$, e então, $R(x) \cap B \neq \varnothing$, fazendo com que seja impossível que $R(x) \subseteq B$. Em ambos os casos, $\mathcal{L}(B)(x) \leq \mathcal{L}(A)(x)$ para $A \subseteq B$, e então, essa classe de testes é monotônica.

Invertibilidade: Verificada no exemplo $\mathbf{3 . 5}$.

Consonância forte da união: Seja $I$ um conjunto de índices arbitrário e $\left\{A_{i}\right\}_{i \in I}, A_{i} \in \sigma(\Theta) \forall i \in I$, tais que $\cup_{i \in I} A_{i} \in \sigma(\Theta)$. Se rejeitarmos todas as hipóteses $A_{i}, R(x) \subseteq A_{i}^{c}$ para todo $i \in I$, e então

$$
R(x) \subseteq \cap_{i \in I} A_{i}^{c}=\left(\cup_{i \in I} A_{i}\right)^{c}
$$

De modo que também rejeitamos $\cup_{i \in I} A_{i}$.

Consonância forte da intersecção: Seja $I$ um conjunto de índices arbitrário e $\left\{A_{i}\right\}_{i \in I}, A_{i} \in \sigma(\Theta) \forall$ $i \in I$, tais que $\cap_{i \in I} A_{i} \in \sigma(\Theta)$. Se aceitarmos todas as hipóteses $A_{i}, R(x) \subseteq A_{i}$ para todo $i \in I$, então

$$
R(x) \subseteq \cap_{i \in I} A_{i}
$$

De modo que também aceitamos $\cap_{i \in I} A_{i}$.

Portanto, essa classe de testes é fortemente consistente.

Dessa forma, para qualquer estimador por região $R$, a classe de testes de hipóteses agnósticos associada a ele será fortemente consistente. Mais do que isso, o próximo resultado nos garante que os únicos testes fortemente consistentes são desse tipo.

Resultado 3.1 (Esteves et al. (2016)). Suponha que $\sigma(\Theta)$ é tal que $\{\theta\} \in \sigma(\Theta)$ para todo $\theta \in \Theta$. Uma classe de testes agnósticos é fortemente consistente se, e somente se, é um teste baseado em um estimador por região (Exemplo 3.5). 
Esse resultado é análogo, num certo sentido, ao visto no capítulo anterior (Resultado 2.1): da mesma forma que nos testes de hipóteses usuais, apresentar coerência lógica no máximo de situações possíveis (representado pelas desideratas D2 e D3) está intimamente ligado a estimação pontual (como discutido em Izbicki (2010) e Silva (2014)), nos testes de hipóteses agnósticos, o resultado acima aponta para uma conexão entre consistência lógica máxima (no contexto das propriedades vistas neste capítulo) e estimação por região, com a condição de ser possível testar todas as hipóteses simples $(\{\theta\} \in \sigma(\Theta), \forall \theta \in \Theta)$, que podem ser em alguns casos precisas.

Testar hipóteses precisas é um problema controverso na inferência estatística, pela aparentemente comum divergência de resultados nas abordagens frequentistas e bayesianas como discutido em Berger e Delampady (1987) e também pela discussão prática existente sobre o quão razoável é testar esse tipo de hipótese, posto que dificilmente conseguimos medir esses parâmetros com precisão suficiente de modo a comprovar essa hipótese na maioria dos casos. Felizmente, nessa classe de testes não temos nenhum dos problemas: no caso de hipóteses unitárias precisas, só aceitaremos quando a estimativa por região for também formada por apenas um ponto e igual a hipótese, o que parece ser razoável, para outros casos de estimativas, o resultado é permanecer agnóstico ou rejeitar a hipótese. Na próxima seção, examinaremos classes de testes de hipóteses agnósticos fortemente consistentes sob a teoria da decisão bayesiana.

\subsection{Resultados Considerando a Teoria da Decisão}

O objetivo dessa seção é apresentar alguns resultados para classes de testes de hipótese agnósticos gerados por famílias de funções de perda (Definição 3.2). Em Esteves et al. (2016), já foram especificadas que restrições devemos impor às famílias de funções de perda para induzir a testes de Bayes agnósticos que atendam individualmente as propriedades de monotonicidade, invertibilidade, consonância fraca com a união e consonância fraca com a intersecção (as propriedades que caracterizam a consistência fraca) para qualquer priori $\pi$, porém, tais condições ainda não foram estudadas para o caso fortemente consistente. A seguir, trazemos a definição de perdas próprias, que será importante ao desenvolver os resultados.

Definição 3.11 (Perdas próprias - Esteves et al. (2016)). Uma família de funções de perda $\left(L_{A}\right)_{A \in \sigma(\Theta)}$ é de perdas próprias se

$$
\begin{cases}L_{A}(0, \theta)<L_{A}\left(\frac{1}{2}, \theta\right)<L_{A}(1, \theta) & \text {, se } \theta \in A \\ L_{A}(0, \theta)>L_{A}\left(\frac{1}{2}, \theta\right)>L_{A}(1, \theta) & , \text { se } \theta \notin A \\ L_{A}\left(\frac{1}{2}, \theta\right)<\frac{L_{A}(0, \theta)+L_{A}(1, \theta)}{2} & , \forall \theta \in \Theta\end{cases}
$$

Ou seja, uma família de funções de perda $\left(L_{A}\right)_{A \in \sigma(\Theta)}$ é de perdas próprias, se a perda é menor ao tomar a decisão correta (rejeitar a hipótese quando ela é falsa ou aceitar quando é verdadeira) do que ao tomar a decisão incorreta (rejeitar a hipótese quando ela é verdadeira ou aceitar quando é falsa), e se, ao permanecermos agnósticos, a perda é menor que a de tomar a decisão incorreta e maior que tomar a decisão correta, com a restrição de que seja sempre menor que a perda esperada ao aleatorizar, com probabilidade igual, a decisão entre rejeitar e aceitar a hipótese. Evidentemente, existem outras famílias de funções de perdas possíveis que podem ser interessantes e que gerariam testes de hipóteses agnósticos de Bayes. O próximo resultado é fundamental para a derivação dos 
dois resultados principais dessa seção.

Resultado 3.2. Seja $\Theta$ finito e $\left(\phi_{A}\right)_{A \in \mathcal{P}(\Theta)}$ uma classe de testes de hipóteses agnósticos fortemente consistente. Temos que:

(i) Para todo $x \in \chi$, existe $\theta_{0} \in \Theta$ tal que $\phi_{\left\{\theta_{0}\right\}}(x)<1$.

(ii) Para todo $x \in \chi$, se existe $\theta_{0} \in \Theta$ tal que $\phi_{\left\{\theta_{0}\right\}}(x)=0$ então $\phi_{\{\theta\}}(x)=1, \forall \theta \neq \theta_{0}$.

Demonstração: (i) Seja $\Theta=\left\{\theta_{1}, \ldots, \theta_{k}\right\}$, suponha que existe $x_{0} \in \chi$ tal que $\phi_{\{\theta\}}\left(x_{0}\right)=1, \forall$ $\theta \in \Theta$. Pela consonância forte da união temos que $\phi_{\Theta}\left(x_{0}\right)=\phi_{\bigcup_{i=1}^{k}\left\{\theta_{i}\right\}}\left(x_{0}\right)=1$. Por outro lado, pela monotonicidade, $\phi_{\varnothing}\left(x_{0}\right)=1$, pois $\varnothing \subset\{\theta\}, \theta \in \Theta$. Logo, $\phi_{\varnothing}\left(x_{0}\right)=\phi_{\Theta}\left(x_{0}\right)=1$, o que significaria que $\left(\phi_{A}\right)_{A \in \mathcal{P}(\Theta)}$ não pode ser invertível.

(ii) Se existe $\theta_{0} \in \Theta$ tal que $\phi_{\left\{\theta_{0}\right\}}(x)=0$, pela invertibilidade, $\phi_{\left\{\theta: \theta \in \Theta, \theta \neq \theta_{0}\right\}}(x)=1$, e pela monotonicidade, $\phi_{\{\theta\}}(x)=1, \forall \theta \in \Theta, \theta \neq \theta_{0}$.

Esse resultado nos diz que, no caso em que o espaço paramétrico e o espaço amostral são finitos, testes de hipóteses agnósticos fortemente consistentes devem aceitar um dos possíveis valores para o parâmetro ou permanecer agnóstico quanto a vários deles, quando avaliamos com respeito aos conjuntos unitários de parâmetros. O próximo resultado discorre sobre a existência de uma classe de testes de hipóteses fortemente consistente dentro da teoria da decisão bayesiana:

Resultado 3.3. Seja $\Theta$ finito, $\mathcal{P}(\Theta)$ o conjunto das partes de $\Theta$, $\chi$ finito $e\left(\phi_{A}\right)_{A \in \mathcal{P}(\Theta)}$ uma classe de testes de hipóteses agnósticos fortemente consistente. Então, existe $\mathbb{P}: \mathcal{P}(\Theta \times \chi) \rightarrow[0,1]$ medida de probabilidade e $\left(L_{A}\right)_{A \in \mathcal{P}(\Theta)}$ uma família de funçôes de perdas próprias tal que $\left(\phi_{A}\right)_{A \in \mathcal{P}(\Theta)}$ é classe de testes de Bayes agnósticos gerada por $\left(L_{A}\right)_{A \in \mathcal{P}(\Theta)}$ (Definiçãa 3.2) contra a $\mathbb{P}$-marginal de $\theta$.

Demonstração: Seja $\Theta=\left\{\theta_{1}, \ldots, \theta_{k}\right\}$. Do resultado 3.2 item (i), temos que:

$$
\sum_{i=1}^{k}\left(1-\phi_{\left\{\theta_{i}\right\}}(x)\right)>0, \quad \forall x \in \chi
$$

Dessa forma, para um $x \in \chi$ fixado, seja $\mu_{x}: \mathcal{P}(\Theta) \rightarrow \mathbb{R}_{+}$dada por:

$$
\mu_{x}(A)=\frac{\sum_{\theta \in A}\left[1-\phi_{\{\theta\}}(x)\right]}{\sum_{\theta \in \Theta}\left[1-\phi_{\{\theta\}}(x)\right]}
$$

Aqui, para $x \in \chi$ existem duas opções:

- Se existe $\theta_{0} \in \Theta$ tal que $\phi_{\left\{\theta_{0}\right\}}(x)=0$, pelo item (ii) do resultado 3.2

$$
\sum_{\theta \in \Theta}\left[1-\phi_{\{\theta\}}(x)\right]=1-\phi_{\left\{\theta_{0}\right\}}(x)=1
$$

Portanto,

$$
\mu_{x}(A)= \begin{cases}1, & \text { se } \theta_{0} \in A \\ 0, & \text { se } \theta_{0} \notin A\end{cases}
$$

Ou seja, $\mu_{x}$ é medida degenerada em $\theta_{0}\left(\theta_{0}(x)\right)$. 
- Se não existe $\theta_{0} \in \Theta$ tal que $\phi_{\left\{\theta_{0}\right\}}(x)=0$, segue, do resultado 3.2 item $\mathbf{i}$, que $\exists \theta^{*} \in \Theta$ tal que $\phi_{\left\{\theta^{*}\right\}}=\frac{1}{2}$. Logo, para $A g(x)=\left\{\theta \in \Theta: \phi_{\{\theta\}}=\frac{1}{2}\right\} \neq \varnothing$

$$
\sum_{i=1}^{k}\left[1-\phi_{\left\{\theta_{i}\right\}}(x)\right]=\frac{1}{2}|A g(x)|
$$

Portanto,

$$
\mu_{x}(A)=\frac{\frac{1}{2}|A g(x) \cap A|}{\frac{1}{2}|A g(x)|}=\frac{|A g(x) \cap A|}{|A g(x)|}
$$

Nesse segundo caso, é fácil ver que $\mu_{x}(A)$ é medida de probabilidade. Tomando $\mu_{0}$ uma distribuição de probabilidade qualquer em $\mathcal{P}(\chi)$, temos que $\mathbb{P}: \mathcal{P}(\Theta \times \chi) \rightarrow \mathbb{R}_{+}$dada por

$$
\mathbb{P}(B)=\sum_{(\theta, x) \in B} \mu_{0}(\{x\}) \mu_{x}(\{\theta\})
$$

é medida de probabilidade em $\mathcal{P}(\Theta \times \chi)$.

Para $A \in \mathcal{P}(\Theta)$, seja $\left(L_{A}\right)_{A \in \mathcal{P}(\Theta)}$ uma família de funções de perda do tipo $L_{A}:\left\{0, \frac{1}{2}, 1\right\} \times \Theta \rightarrow \mathbb{R}_{+}$ que gera a classe de testes de hipóteses agnósticos de Bayes (Definição 3.2) $\left(\phi_{A}^{*}\right)_{A \in \mathcal{P}(\Theta)}$ dada por:

$$
\phi_{A}^{*}(x)=\left\{\begin{array}{ll}
0, & \text { se } \mathbb{P}(\theta \in A \mid x)>1-\delta \\
1, & \text { se } \mathbb{P}(\theta \in A \mid x)<\delta \\
\frac{1}{2}, & \text { se } \mathbb{P}(\theta \in A \mid x) \in[\delta, 1-\delta]
\end{array}, \text { com } 0<\delta<\frac{1}{k}=\frac{1}{|\Theta|}\right.
$$

Vamos verificar que $\left(\phi_{A}^{*}\right)_{A \in \mathcal{P}(\Theta)}$ coincide com a classe de testes de hipóteses agnósticos fortemente consistente $\left(\phi_{A}\right)_{A \in \mathcal{P}(\Theta)}$, gerada pelo estimador por região $R(x)=\left\{\theta \in \Theta: \phi_{\{\theta\}}(x)<1\right\}$, dada por:

$$
\phi_{A}(x)= \begin{cases}0, & \text { se } R(x) \subseteq A \\ 1, & \text { se } R(x) \subseteq A^{c} \\ \frac{1}{2}, & \text { se } R(x) \cap A \neq \varnothing \text { e } R(x) \cap A^{c} \neq \varnothing\end{cases}
$$

Temos que:

$$
\phi_{A}(x)=1 \Longleftrightarrow R(x) \subseteq A^{c} \Longleftrightarrow R(x) \cap A=\varnothing
$$

Aqui temos 2 casos:

- Se existe $\theta_{0} \in \Theta$ tal que $\phi_{\left\{\theta_{0}\right\}}(x)=0$ :

$$
\begin{gathered}
R(x) \cap A=\varnothing \Longleftrightarrow \theta_{0} \in A^{c} \Longleftrightarrow \mathbb{P}(\theta \in A \mid x)=\mu_{x}(A)=0 \Longleftrightarrow\left(\mu_{x}(A) \text { degenerada em } \theta_{0}\right) \\
\Longleftrightarrow P(\theta \in A \mid x)<\delta \Longleftrightarrow \phi_{A}^{*}(x)=1
\end{gathered}
$$

- Se não existe $\theta_{0} \in \Theta$ tal que $\phi_{\left\{\theta_{0}\right\}}(x)=0$ :

$$
\begin{gathered}
R(x) \cap A=\varnothing \Longleftrightarrow \mathbb{P}(\theta \in A \mid x)=\mu_{x}(A)=\frac{|A g(x) \cap A|}{|A g(x)|}=0 \Longleftrightarrow(0<\delta<1 / k) \\
\Longleftrightarrow P(\theta \in A \mid x)<\delta \Longleftrightarrow \phi_{A}^{*}(x)=1
\end{gathered}
$$


Alem disso:

$$
\begin{gathered}
\phi_{A}(x)=\frac{1}{2} \Longleftrightarrow R(x) \cap A \neq \varnothing \text { e } R(x) \cap A^{c} \neq \varnothing \Longleftrightarrow(|R(x)| \geq 2) \\
\Longleftrightarrow|A g(x) \cap A| \in\{1, \ldots,|A g(x)|-1\} \Longleftrightarrow \mathbb{P}(\theta \in A \mid x)=\mu_{x}(A) \in\left\{\frac{1}{|A g(X)|}, \ldots, \frac{|A g(X)|-1}{|A g(X)|}\right\} \Longleftrightarrow \\
\Longleftrightarrow \delta \leq \mathbb{P}(\theta \in A \mid x) \leq 1-\delta \Longleftrightarrow \phi_{A}^{*}(x)=\frac{1}{2}
\end{gathered}
$$

E finalmente:

$$
\phi_{A}(x)=0 \Longleftrightarrow R(x) \subseteq A
$$

Aqui novamente precisamos avaliar os dois casos possíveis:

- Se existe $\theta_{0} \in \Theta$ tal que $\phi_{\left\{\theta_{0}\right\}}(x)=0$ :

$$
\phi_{A}(x)=0 \Longleftrightarrow R(x)=\left\{\theta_{0}\right\} \subseteq A \Longleftrightarrow \mathbb{P}(\theta \in A \mid x)=\mu_{x}(A)=1 \Longleftrightarrow \phi_{A}^{*}(x)=0
$$

- Se não existe $\theta_{0} \in \Theta$ tal que $\phi_{\left\{\theta_{0}\right\}}(x)=0$ :

$$
\begin{gathered}
\phi_{A}(x)=0 \Longleftrightarrow R(x)=A g(x) \subseteq A \Longleftrightarrow \\
\Longleftrightarrow \mathbb{P}(\theta \in A)=\mu_{x}(A)=\frac{|A g(x) \cap A|}{|A g(x)|}=\frac{|A g(x)|}{|A g(x)|}=1 \Longleftrightarrow \phi_{A}^{*}(x)=0
\end{gathered}
$$

Concluímos então que, $\left(\phi_{A}^{*}\right)_{A \in \mathcal{P}(\Theta)}$ coincide $\operatorname{com}\left(\phi_{A}\right)_{A \in \mathcal{P}(\Theta)}$.

Portanto, verificamos que uma classe de testes de hipóteses agnósticos fortemente consistente é compatível com algum agente decisor, pois existe uma distribuição a priori e uma família de funções de perda que resulta em uma classe de testes de Bayes que atende as propriedades. Esse resultado é análogo ao visto no capítulo anterior para os testes de hipóteses usuais (Resultado 2.2). Vale ressaltar que o resultado $\mathbf{3 . 3}$ pode ser obtido com outras construções para $\mathbb{P}$ e outras funções de perda.

O próximo resultado complementa o anterior, concluíndo que se um teste fortemente consistente, baseado em um estimador por região, é de Bayes, então esse estimador por região é também de Bayes contra alguma perda.

Resultado 3.4. Seja $\Theta$ finito, $\chi$ finito, $\left(\phi_{A}\right)_{A \in \mathcal{P}(\Theta)}$ uma classe de testes de hipóteses agnósticos fortemente consistente gerada por $R: \chi \rightarrow \mathcal{P}(\Theta) \backslash\{\varnothing\}$. Se existe priori $\pi$ para $\theta$ e familia de funçôes de perdas próprias $\left(L_{A}\right)_{A \in \mathcal{P}(\Theta)}, L_{A}:\left\{0, \frac{1}{2}, 1\right\} \times \Theta \rightarrow \mathbb{R}^{+}$que geram $\left(\phi_{A}\right)_{A \in \mathcal{P}(\Theta)}$ contra $\pi$, então existe $\bar{L}: \mathcal{P}(\Theta) \backslash\{\varnothing\} \times \Theta \rightarrow \mathbb{R}$ tal que $R$ é estimador por região de Bayes para $\theta$ contra $\pi$ considerando $\bar{L}$.

Demonstração: Para cada $A \in \mathcal{P}(\Theta)$ e $\theta \in \Theta$ (finito), seja

$$
\bar{L}(A, \theta)=\sum_{\theta^{\prime} \in A}\left[L_{\left\{\theta^{\prime}\right\}}\left(\frac{1}{2}, \theta\right)-L_{\left\{\theta^{\prime}\right\}}(1, \theta)\right]
$$

Para $x \in \chi$, consideraremos as duas possibilidades para $R(X)$. Se $|R(x)|>1$, é claro que: 
- Não existe $\theta_{0} \in \Theta$ tal que $\phi_{\left\{\theta_{0}\right\}}(x)=0$.

- Para cada $\theta \in R(x), \phi_{\{\theta\}}(x)=\frac{1}{2}$.

- Para cada $\theta \notin R(x), \phi_{\{\theta\}}(x)=1$

Como $\left(\phi_{A}\right)_{A \in \mathcal{P}(\Theta)}$ é gerada por $\left(L_{A}\right)_{A \in \mathcal{P}(\Theta)}$, segue que para $\theta^{\prime} \in R(x)$ :

$$
\mathbb{E}_{\theta \mid x}\left[L_{\left\{\theta^{\prime}\right\}}\left(\frac{1}{2}, \theta\right) \mid x\right]<\mathbb{E}_{\theta \mid x}\left[L_{\left\{\theta^{\prime}\right\}}(1, \theta) \mid x\right]
$$

E para $\theta^{\prime} \notin R(x)$ :

$$
\mathbb{E}_{\theta \mid x}\left[L_{\left\{\theta^{\prime}\right\}}(1, \theta) \mid x\right]<\mathbb{E}_{\theta \mid x}\left[L_{\left\{\theta^{\prime}\right\}}\left(\frac{1}{2}, \theta\right) \mid x\right]
$$

Verificaremos que $R(x)$ é de Bayes considerando $\bar{L}$ contra $\pi$. Para $A \in \mathcal{P}(\Theta) \backslash\{\varnothing\}$, temos:

$$
\begin{aligned}
\mathbb{E}_{\theta \mid x}[\bar{L}(A, \theta) \mid x]= & \sum_{\theta \in \Theta} \bar{L}(A, \theta) \pi(\theta \mid x)=\sum_{\theta \in \Theta}\left[\sum_{\theta^{\prime} \in A}\left[L_{\left\{\theta^{\prime}\right\}}\left(\frac{1}{2}, \theta\right)-L_{\left\{\theta^{\prime}\right\}}(1, \theta)\right] \pi(\theta \mid x)=\right. \\
= & \sum_{\theta^{\prime} \in A}\left[\sum_{\theta \in \Theta}\left[L_{\left\{\theta^{\prime}\right\}}\left(\frac{1}{2}, \theta\right)-L_{\left\{\theta^{\prime}\right\}}(1, \theta)\right] \pi(\theta \mid x)\right]= \\
& \sum_{\theta^{\prime} \in A}\left[\sum_{\theta \in \Theta}\left[L_{\left\{\theta^{\prime}\right\}}\left(\frac{1}{2}, \theta\right) \pi(\theta \mid x)-L_{\left\{\theta^{\prime}\right\}}(1, \theta) \pi(\theta \mid x)\right]\right]= \\
= & \sum_{\theta^{\prime} \in A}\left[\sum_{\theta \in \Theta} L_{\left\{\theta^{\prime}\right\}}\left(\frac{1}{2}, \theta\right) \pi(\theta \mid x)-\sum_{\theta \in \Theta} L_{\left\{\theta^{\prime}\right\}}(1, \theta) \pi(\theta \mid x)\right]= \\
& \sum_{\theta^{\prime} \in A}\left[\mathbb{E}_{\theta \mid x}\left[L_{\left\{\theta^{\prime}\right\}}\left(\frac{1}{2}, \theta\right) \mid x\right]-\mathbb{E}_{\theta \mid x}\left[L_{\left\{\theta^{\prime}\right\}}(1, \theta) \mid x\right]\right]
\end{aligned}
$$

Do fato de que a família $\left(L_{A}\right)_{A \in \mathcal{P}(\Theta)}$ é de perdas próprias:

- $\mathbb{E}_{\theta \mid x}\left[L_{\left\{\theta^{\prime}\right\}}\left(\frac{1}{2}, \theta\right) \mid x\right]-\mathbb{E}_{\theta \mid x}\left[L_{\left\{\theta^{\prime}\right\}}(1, \theta) \mid x\right]<0, \forall \theta^{\prime} \in R(X)$

- $\mathbb{E}_{\theta \mid x}\left[L_{\left\{\theta^{\prime}\right\}}(1, \theta) \mid x\right]-\mathbb{E}_{\theta \mid x}\left[L_{\left\{\theta^{\prime}\right\}}\left(\frac{1}{2}, \theta\right) \mid x\right]>0, \forall \theta^{\prime} \notin R(X)$

E então:

$$
\begin{gathered}
\sum_{\theta^{\prime} \in A}\left[\mathbb{E}_{\theta \mid x}\left[L_{\left\{\theta^{\prime}\right\}}\left(\frac{1}{2}, \theta\right) \mid x\right]-\mathbb{E}_{\theta \mid x}\left[L_{\left\{\theta^{\prime}\right\}}(1, \theta) \mid x\right]\right] \\
\geq \sum_{\theta^{\prime} \in R(x)}\left[\mathbb{E}_{\theta \mid x}\left[L_{\left\{\theta^{\prime}\right\}}\left(\frac{1}{2}, \theta\right) \mid x\right]-\mathbb{E}_{\theta \mid x}\left[L_{\left\{\theta^{\prime}\right\}}(1, \theta) \mid x\right]\right]=\mathbb{E}_{\theta \mid x}[\bar{L}(R(x), \theta) \mid x]
\end{gathered}
$$

Logo, $\forall A \in \mathcal{P}(\Theta) \backslash\{\varnothing\}$ :

$$
\mathbb{E}_{\theta \mid x}[\bar{L}(A, \theta) \mid x] \geq \mathbb{E}_{\theta \mid x}[\bar{L}(R(x), \theta) \mid x]
$$

Para $|R(x)|=1$ :

- Existe $\theta_{0} \in \Theta$ tal que $\phi_{\left\{\theta_{0}\right\}}=0$

- $R(x)=\left\{\theta_{0}\right\}$ 
- Para cada $\theta \notin R(x), \phi_{\{\theta\}}(x)=1$

Assim, para $\theta^{\prime} \neq \theta_{0}$ :

$$
\mathbb{E}_{\theta \mid x}\left[L_{\left\{\theta^{\prime}\right\}}(1, \theta) \mid x\right]<\mathbb{E}_{\theta \mid x}\left[L_{\left\{\theta^{\prime}\right\}}\left(\frac{1}{2}, \theta\right) \mid x\right]
$$

Para $\theta=\theta_{0}$, vale que:

$$
\mathbb{E}_{\theta \mid x}\left[L_{\left\{\theta_{0}\right\}}(0, \theta) \mid x\right] \leq \mathbb{E}_{\theta \mid x}\left[L_{\left\{\theta_{0}\right\}}(1, \theta) \mid x\right]
$$

Como $\left(L_{A}\right)_{A \in \mathcal{P}(\Theta)}$ é própria e $\Theta$ é finito, resulta que

$$
\mathbb{E}_{\theta \mid x}\left[L_{\left\{\theta_{0}\right\}}\left(\frac{1}{2}, \theta\right) \mid x\right]<\mathbb{E}_{\theta \mid x}\left[L_{\left\{\theta_{0}\right\}}(1, \theta) \mid x\right]
$$

Procedendo como no caso $|R(x)|>1$, segue, de $(*)$ e $(* *)$, que, $\forall A \in \mathcal{P}(\Theta) \backslash\{\varnothing\}$

$$
\mathbb{E}_{\theta \mid x}[\bar{L}(A, \theta) \mid x] \geq \mathbb{E}_{\theta \mid x}\left[\bar{L}\left(\left\{\theta_{0}\right\}, \theta\right) \mid x\right]=\mathbb{E}_{\theta \mid x}[\bar{L}(R(x), \theta) \mid x]
$$

Em qualquer caso, $R(x)$ é estimativa de Bayes por região para $\theta$ contra $\pi$ considerando a função de perda $\bar{L}$.

Novamente, de forma análoga ao resultado $\mathbf{2 . 3}$ visto no capítulo anterior, da mesma forma que testes de hipóteses usuais logicamente consistentes de Bayes estão ligados a um estimador pontual de Bayes, agora, testes de hipóteses agnósticos logicamente consistentes de Bayes estão ligados a um estimador por região de Bayes. Em outras palavras, se um teste de hipótese agnóstico logicamente consistente é de Bayes, o estimador por região associado não pode ser qualquer estimador, ele é também de Bayes contra alguma perda específica. 


\section{Capítulo 4}

\section{Conclusões}

\subsection{Considerações Finais}

Neste trabalho, discutimos algumas possíveis formas de inconsistências lógicas ao realizar testes de hipóteses simultâneos, revisando propriedades avaliadas em estudos anteriores que caracterizam testes logicamente consistentes. Trazemos como alternativa os testes de hipóteses agnósticos, como formulados em Esteves et al. (2016), que permitem para uma hipótese, além de aceitar ou rejeitar, a opção de suspender o julgamento, permanecendo agnóstico sobre a compatibilidade ou não dos nossos dados com a nossa hipótese. Para essa alternativa, trouxemos as devidas adaptações que devem ser feitas nas propriedades lógicas revistas. Também realçamos o impacto de exigir as diversas propriedades simultaneamente e as possíveis relações entre elas tanto para os testes de hipótese usuais quanto para os agnósticos.

Finalmente, apresentamos alguns resultados para os testes de hipóteses agnósticos logicamente consistentes sob a ótica da teoria da decisão bayesiana: no primeiro resultado, mostramos que testes fortemente consistentes são compatíveis com algum agente decisor bayesiano em espaços finitos; no segundo resultado, verificamos que esses testes, quando são de Bayes, são baseados em um estimador por região que também é de Bayes, contra alguma perda específica.

\subsection{Perspectivas Futuras}

- Estudar a existência de famílias de funções de perdas que garantam consistência lógica contra qualquer priori.

- Estudar a relação entre testes de hipóteses agnósticos e o problema de definição de tamanho amostral.

- Explorar se restrições impostas à $\sigma$-álgebra de $\Theta$ podem ampliar o conjunto de testes possíveis que atendam a consistência forte.

- Estudar os testes de hipóteses agnósticos quando utilizados em hipóteses precisas.

- Estudar a extensão dos resultados obtidos, considerando os casos em que o espaço paramétrico é enumerável ou infinito, para distribuições contínuas ou com outras famílias de funções de perda. 
- Estudar condições, se possível, para que testes baseados em regiões HPD, que são fortemente consistentes, sejam também testes de Bayes, sob a teoria da decisão bayesiana. 


\section{Referências Bibliográficas}

$\operatorname{Berg}(\mathbf{2 0 0 4})$ N. Berg. No-decision classification: An alternative to testing for statistical significance. The Journal of Socio-Economics, 33(5):631-650. Citado na pág. 11

Berger e Delampady(1987) J. O. Berger e M. Delampady. Testing precise hypotheses. Statistical Science, 2(5):317-335. Citado na pág. 20

Betz e Levin(1982) M. A. Betz e J. R Levin. Coherent analysis-of-variance hypothesis-testing strategies: A general model. Journal of Educational Statistics, 7(3):193-206. Citado na pág. 3

Chow(1970) C. Chow. On optimum recognition error and reject tradeoff. IEEE Transactions on information theory, 16(1):41-46. Citado na pág. 11

Coscrato et al.(2018) V. Coscrato, R. Izbicki e R. B. Stern. Agnostic tests can control the type i and type ii errors simultaneously. arXiv preprint arXiv:1805.04620. Citado na pág. 11

El-Yaniv e Wiener(2010) R. El-Yaniv e Y. Wiener. On the foundations of noise-free selective classification. Journal of Machine Learning Research, 11:1605-1641. Citado na pág. 11

Esteves et al.(2016) L. G. Esteves, R. Izbicki, J. M. Stern e R. B. Stern. The logical consistency of simultaneous agnostic hypothesis tests. Entropy, 18(7):256. Citado na pág. 11, 12, 13, 14, 15, 16, $17,18,19,20,27$

Fossaluza(2008) V. Fossaluza. Testes de hipóteses em eleiçoes majoritárias. Dissertação de Mestrado, Universidade de São Paulo. Citado na pág. 3, 4

Fossaluza et al.(2017) V. Fossaluza, R. Izbicki, G. M. Silva e L. G. Esteves. Coherent hypothesis testing. The American Statistician, 71(3):242-248. Citado na pág. 12

Gabriel(1969) K. R. Gabriel. Simultaneous test procedures-some theory of multiple comparisons. The Annals of Mathematical Statistics, 40(1):224-250. Citado na pág. 3, 4

Hommel e Bretz(2008) G. Hommel e F. Bretz. Aesthetics and power considerations in multiple testing - a contradiction? Biometrical Journal, 50(5):657-666. Citado na pág. 3

Izbicki(2010) R. Izbicki. Classes de testes de hipóteses. Dissertação de Mestrado, Universidade de São Paulo. Citado na pág. 1, 3, 4, 5, 6, 7, 8, 9, 20

Kendall e Stuart(1961) M. Kendall e A. Stuart. The advanced theory of statistics. Vol.2: Inference and relationship. Hafner Publishing Company. Citado na pág. 1, 4

Kruskal(1978) W. H. Kruskal. Tests of significance. International Encylopedia of Social Sciences, 2:944-958. Citado na pág. 4

Lavine e Schervish(1999) M. Lavine e M. J. Schervish. Bayes factors: What they are and what they are not. The American Statistician, 53(2):119-122. Citado na pág. 14

Lehmann e Romano(2005) E. L. Lehmann e J. P. Romano. Testing Statistical Hypotheses. Springer Science \& Business Media. Citado na pág. 1 
Lindgren(1993) B. W. Lindgren. Statistical Theory. Chapman and Hall. Citado na pág. 4, 12

Lindley(1970) D. V. Lindley. Introduction to Probability and Statistics from a Bayesian Viewpoint, Part 2, Inference. Cambridge University Press. Citado na pág. 7

Nicolau(1993) J. M. Nicolau. Sistema Eleitoral e Reforma Política. Foglio Editora. Citado na pág. 1

Pereira e Stern(1999) C. A. B. Pereira e J. M. Stern. Evidence and credibility: Full bayesian significance test for precise hypotheses. Entropy, 1(4):99-110. Citado na pág. 4, 12

Robert(2007) C. Robert. The Bayesian Choice: From Decision-Theoretic Foundations to Computational Implementation. Springer Science \& Business Media. Citado na pág. 5, 12, 14

Rom e Holland(1995) D. M. Rom e B. Holland. A new closed multiple testing procedure for hierarchical families of hypotheses. Journal of statistical planning and inference, 46(3):265-275. Citado na pág. 3

Royall(1997) R. M. Royall. Statistical Evidence: A likelihood paradigm. Chapman and Hall. Citado na pág. 5

Schervish(1996) M. J. Schervish. P values: What they are and what they are not. The American Statistician, 50(3):203-206. Citado na pág. 12

Schervish(1995) M. J. Schervish. Theory of Statistics. Springer Series in Statistics. Springer New York. Citado na pág. 6, 13

Silva(2010) G. M. Silva. Monotonicidade em testes de hipóteses. Dissertação de Mestrado, Universidade de São Paulo. Citado na pág. 3, 4

Silva(2014) G. M. Silva. Propriedades lógicas de classes de testes de hipóteses. Tese de Doutorado, Universidade de São Paulo. Citado na pág. 3, 4, 5, 6, 7, 8, 9, 20 